\title{
Electrochemical and Quantum Chemical Studies on Corrosion Inhibition Performance of 2,2'-(2-Hydroxyethylimino)bis[N-(alphaalpha-dimethylphenethyl)-N-methylacetamide] on Mild Steel Corrosion in 1M HCl Solution
}

\author{
Iman Danaee ${ }^{a} *$ (D), S. RameshKumar ${ }^{b}$, M. RashvandAveic, M. Vijayan ${ }^{d}$ \\ ${ }^{a}$ Petroleum University of Technology, Abadan Faculty of Petroleum Engineering, Abadan, Iran \\ ${ }^{b}$ Sri Vasavi College, Department of Chemistry, Erode, Tamilnadu-638 316, India. \\ ${ }^{c} K$. N. Toosi University of Technology, Department of chemistry, Tehran, Iran \\ ${ }^{d}$ Central Electrochemical Research Institute, Centre for Conducting Polymers, Electrochemical \\ Materials Science Division, Karikudi, 630006, India
}

Received: September 7, 2018; Revised: January 13, 2020; Accepted: March 16, 2020

\begin{abstract}
The inhibitory effect of Oxethazaine drug, 2,2'-(2-Hydroxyethylimino)bis[N-(alphaalphadimethylphenethyl)-N-methylacetamide] on corrosion of mild steel in $1 \mathrm{M} \mathrm{HCl}$ solution was studied by weight loss measurements, electrochemical impedance spectroscopy and potentiodynamic polarization methods. The results of gravimetric and electrochemical methods demonstrated that the inhibition efficiency increased with an increase in inhibitor concentration in $1 \mathrm{M} \mathrm{HCl}$ solution. The results from electrochemical impedance spectroscopy proved that the inhibition action of this drug was due to adsorption on the metal surface. Potentiodynamic polarization studies revealed that the molecule was a mixed type inhibitor. The adsorption of the molecule on the metal surface was found to obey Langmuir Adsorption isotherm. Potential of zero charge at the metal-solution interface was measured to provide the inhibition mechanism. The temperature dependence of the corrosion rate was also studied in the temperature range from 30 to $50^{\circ} \mathrm{C}$. Quantum chemical calculations were applied to correlate electronic structure parameters of the drug with its inhibition performance. The obtained theoretical results have been adapted with the experimental data.
\end{abstract}

Keywords: Corrosion inhibitors; Potential of zero charge; Impedance; Quantum chemical calculations.

\section{Introduction}

The surface of iron and steel, existing in many forms ranging from exposed metal to oxidized to different degrees, finds many industrial applications ${ }^{1-3}$. Recent investigations have emphasized the importance for the protection of the metal surfaces in various applications. The spontaneous destruction of metals, starting from their surfaces, due to the corrosive attack of environment brings the undesirable changes at the surfaces and reduces their lifetime, strength and changes the desirable properties of surfaces. In some cases the corrosion products formed are toxic. An accurate description of surface not only helps to identify the prevailing form of corrosion but also the prescription of appropriate anti-corrosion measures. Acid solutions, in general $\mathrm{HCl}$ solutions are widely used in industries for many purposes, such as acid pickling, industrial acid cleaning, acid descaling and oil well acidizing ${ }^{4-7}$. Due to the general aggressive nature of acid solutions, the corrosive attack will be severe ${ }^{4}$ and bring undesirable changes at the surface of metals. Chemical inhibitors are often used to control the corrosive attack and acid consumptions of environment ${ }^{8,9}$. Most of the well-known acid corrosion inhibitors are the organic compound containing $\mathrm{N}, \mathrm{S}, \mathrm{O}$ and $\mathrm{P}$ atoms ${ }^{10,11}$. These organic compounds reduce the metal dissolution by the absorption on the metal surfaces ${ }^{12}$. The adsorption and inhibition efficiency

*e-mail: danaee@put.ac.ir of these compounds greatly depend on the electron density around the hetero atoms, the number of adsorption active centers in the molecule and their surface charge density, molecular size, mode of adsorption and formation of metallic complexes ${ }^{10-21}$. However, the choice of inhibitors are based on the two considerations, first economic consideration and second the presence of the electronegative atoms such as $\mathrm{N}, \mathrm{O}$ in the relatively long compounds. Thermodynamic model is an important tool used for analyzing the corrosion inhibition mechanism of inhibitors and their adsorption on the metal surface ${ }^{14,15}$. Using the type of adsorption isotherm that the molecule follows for the adsorption on mild steel surface, the values of thermodynamic parameters such as adsorption equilibrium constant $\left(K_{\text {ads }}\right)$ and free energy change of adsorption $\left(\Delta \mathrm{G}_{\mathrm{ads}}\right)$ can be calculated and used for predicting the adsorption and inhibition mechanism.

Many authors generally agree that drugs are the good corrosion inhibitors that can complete favorably with green corrosion inhibitor. Moreover, most drugs can be synthesized from natural products. The reasons for using drugs as corrosion inhibitors are; (1) drug molecule contains oxygen, nitrogen and sulphur as active centers, (2) drugs are reported environmentally friendly and important in biological reactions ${ }^{22,23}$. In modern scenario, development of novel biodegradable and less toxic corrosion inhibitors is gaining importance. Biologically active molecules like sulfadimidine, sulfamethoxazole, cefatrexyl, 
apart from other antibacterial and antifungal drugs have been reported as good corrosion inhibitors ${ }^{24-26}$. The use of drugs as corrosion inhibitors for metals in different aggressive environments is widely reported. These include the use of sulpha $\operatorname{drugs}^{27,28}$, antimalerial drugs ${ }^{29}$ and analgesic drugs ${ }^{30}$ as efficient corrosion inhibitors for metals in various media. Moreover, many expired drugs have been reported to be very effective corrosion inhibitors for the protection of mild steel in acidic media $^{31-36}$. This area of research is much important because in addition to being environmentally friendly and ecologically acceptable, expired pharmaceutical drugs are inexpensive, readily available and prevent pollution. On the other hand, deactivation of this expired drug is generally carried out with the risk of air pollution with toxic compounds containing N, S, P or halogen atoms. The expiry date is merely an assurance from the manufacturer that the drug is expected to retain at least $90 \%$ of its original efficacy up to that time and its active substance degrades only negligibly. This type of research can solve two major environmental and economical problems: limitation of environmental pollution with pharmaceutically compounds and reduction of the disposal costs of expired drugs. Thus, this area would give effective nonhazardous alternatives to toxic corrosion inhibitors.

Recently, the research activities are centered on the development of cheaper and greener corrosion inhibitors. In this context, the application of expired drugs as corrosion inhibitors can satisfy both environmental and economic provisos of green chemistry. Dohare et al. on 2017 have used expired Tramadol drug as inhibitor for mild steel corrosion in hydrochloric acid ${ }^{35}$. They employed DFT and experimental studies on the inhibition potentials of expired drug and the inhibition efficiency about $96 \%$ was achieved. Anaee et al. on 2019 studied expired Etoricoxib as a corrosion inhibitor for steel in acidic solution ${ }^{36}$. Expired Etoricoxib indicated high inhibition efficiency in different temperatures.

Density functional theory (DFT) has become a useful theoretical method to interpret the experimental results, enabling one to obtain various structural parameters, even the inhibitor is a larger complex molecule, i.e. DFT links some traditional empirical concepts with quantum mechanical results $^{37,38}$. Therefore, DFT is a connecting bridge between the experimental results and theoretical structural parameters of inhibitor molecule.

The present work deals with the corrosion inhibition nature of Oxethazaine drug, 2,2'-(2-Hydroxyethylimino)bis[N(alphaalpha-dimethylphenethyl)-N-methylacetamide] in the acid medium utilizing; weight loss measurements, the polarization method and electrochemical impedance spectroscopy (EIS). Potential of charge (PZC) was also determined to establish the inhibition mechanism. Quantum chemical calculations have been performed, and several quantum chemical indices are calculated and correlated with the corrosion inhibition nature of the inhibitor in $1 \mathrm{M} \mathrm{HCl}$ medium.

\section{Experimental}

\subsection{Materials}

Mild steel specimens of dimensions $2.5 \mathrm{~cm} \times 1.0 \mathrm{~cm} \times 0.1 \mathrm{~cm}$ with a composition $0.087 \% \mathrm{C}, 0.039 \% \mathrm{mn}, 0.03 \% \mathrm{P}, 0.02 \% \mathrm{~S}$ and rest iron were used for weight loss studies. The specimens were mechanically polished using emery sheets of grade $1 / 0,2 / 0,3 / 0,4 / 0$, and $5 / 0$ and thoroughly washed with double distilled water. Finally the specimens were degreased with acetone and dried. A Teflon coated cylindrical mild steel rod, having an exposed area of $0.02826 \mathrm{~cm}^{2}$, with previously mentioned composition was used for electrochemical studies. Solutions of $1 \mathrm{M} \mathrm{HCl}$ with and without inhibitor were prepared from A.R. grade reagents using double distilled water. $3 \%$ ethanol by volume was added for solubility reasons. The inhibitor purchased from Guide Chem (Analytical Grade), was used as received without further purification. The structure of the inhibitor molecule is shown in Figure 1.

\section{2 Methods}

\subsubsection{Weight loss measurements}

Weight loss measurements were carried out according to the American Society for testing and Materials (ASTM) standard procedure G 31-72. The mild steel specimens in triplicate were immersed for the period of 2 hours in $100 \mathrm{~mL}$ of corrosive media with and without the inhibitor at room temperature $(300 \pm 1 \mathrm{~K})$. The average weight loss of these specimens was used to calculate the inhibition efficiency employing the formula:

$$
I E \%=-\left(\frac{W-W^{\prime}}{W}\right) \times 100
$$

where $W$ and $W^{\prime}$ represent the weight losses in the uninhibited and inhibited solutions respectively. The relative difference between the replica experiments was found to be less than $3 \%$ at room temperature, which shows a good reproducibility.

\subsubsection{Electrochemical measurements}

The impedance measurements were performed using a computer-controlled potentiostat (model Solartron ECI-1286) and the data were analyzed using frequency response analyzer (Solartron FRA-1286). A three electrode set up was used for the electrochemical impedance measurements with a Pt counter electrode and a saturated calomel electrode (SCE) as the reference electrode. A Teflon coated mild steel cylinder, with surface prepared as described in the weight loss experimental method, served as the working electrode. The measurements were carried out in the frequency range $100 \mathrm{kHz}$ to $0.01 \mathrm{~Hz}$ at the open circuit potential by super imposing a sinusoidal AC signal of small amplitude $(10 \mathrm{mV})$. The time interval of 25-30 minutes was given for steady state attainment of open circuit potential. The electrochemical impedance parameters such as double layer capacitance $\left(C_{\mathrm{dl}}\right)$ and polarization resistance $\left(R_{\mathrm{p}}\right)$ were obtained from the Nyquist plots as described elsewhere.

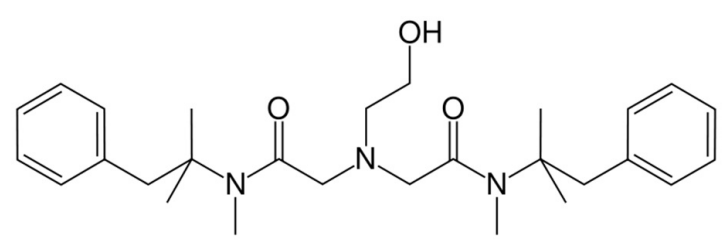

Figure 1. Chemical structure of Oxethazaine drug, (2,2'-(2-Hydroxyethylimino)bis[N-(alphaalpha-dimethyl phenethyl)$\mathrm{N}$-methylacetamide]. 
The potentiodynamic polarization curves were recorded after carrying out the electrochemical impedance measurements, using the same cell setup employed in the impedance measurement, at the potential sweep rate $1.67 \mathrm{mV} \mathrm{s}^{-1}$ from $-700 \mathrm{mV}$ to $-200 \mathrm{mV}$ vs. SCE. The inhibition efficiencies were calculated using the relationship.

$$
I E \%=-\left(\frac{i_{\text {corr }}-i_{\text {corr }}{ }^{\prime}}{i_{\text {corr }}}\right) \times 100
$$

Where $I_{\text {corr }}$ and $I^{\prime}$ corr are the corrosion current densities in the absence and presence of the inhibitor in $1 \mathrm{M} \mathrm{HCl}$ solutions, respectively. The electrochemical impedance spectra were recorded at the $200 \mathrm{~Hz}$ applied single AC frequency at different applied DC potentials and the double layer capacitance values obtained were plotted against the applied DC potentials to determine PZC. All the measurements were taken at room temperature $\left(27 \pm 1^{\circ} \mathrm{C}\right)$.

\subsubsection{Scanning electron microscopy}

The surface morphology of mild steel specimen was analyzed after immersing in the blank and inhibited $\mathrm{HCl}$ solution by Scanning Electron Microscopy (Model-JEOLJSM-6390). The surface morphological characterization was carried out at the magnification $2.0 \mathrm{KX}$ operated at an accelerating voltage $15 \mathrm{kV}$.

\subsubsection{Quantum chemical calculations}

All quantum chemical calculations were performed with complete geometry optimization using the Gaussian 98 code. The molecular sketches of the inhibitor are drawn using Gauss View 3.0. Geometry optimizations were accomplished by two different methods; ab initio methods at the Hartree-Fock (HF) level with the 3-21G and 6-31G(d,p) basis sets and at the density functional theory (DFT) level with the non-local hybrid density functional B3LYP, combining Becke's three-parameter hybrid exchange functional with the correlation functional of Lee et al.at the basis sets $3-21 \mathrm{G}$ and $6-31 \mathrm{G}(\mathrm{d}, \mathrm{p})^{39}$. After the geometry optimization, vibrational analysis was performed and the resulting geometry was checked with respect to true minima on the potential energy surface, as shown by the absence of imaginary frequencies ${ }^{40}$.

The quantum chemical investigations were used to explain the results obtained in gravimetric and electrochemical studies on the corrosion inhibition property of 2,2'-(2-Hydroxyethylimino) bis[N-(alphaalpha-dimethylphenethyl)-N-methylacetamide], looking for good theoretical parameters to characterize its inhibition performance. Statistical analyses were performed using SPSS program version 15.0 for windows. Non-linear regression analyses were performed by unconstrained sum of squared residuals for loss function and the estimation methods of Levenberg-Marquardt using SPSS program version 15.0 for windows.

\section{Results and Discussion}

\subsection{Weight loss measurements}

Weight loss measurements provide the most reliable results concerning the inhibition efficiency of the compounds ${ }^{41}$. The variation of weight loss of mild steel specimens in $1 \mathrm{M} \mathrm{HCl}$ solutions in the absence and presence of the inhibitor is shown in Table 1. It is apparent that the inhibition efficiency increases with an increase in inhibitor concentration in $1 \mathrm{M} \mathrm{HCl}$ solutions. The decrease in corrosion rate in the presence of inhibitor is due to the strong interaction of the inhibitor molecules with the metal surface which lead to forming protective film by the adsorbed inhibitor on the metal surface, separating it from corrosion medium ${ }^{42,43}$. The extent of adsorption increases with an increase in the inhibitor concentration and the maximum adsorption was found at $200 \mathrm{ppm}$. The corrosion suppression ability of the inhibitor might be originated from its tendency to form either strong or weak chemical bonds, with Fe atoms on the metal surface, using the lone pair of electrons present on the $\mathrm{O}$ and $\mathrm{N}$ atoms and the $\pi$ electrons in the benzene ring.

\subsection{Electrochemical impedance spectroscopy}

The impedance spectra obtained for the dissolution of mild steel in $1 \mathrm{M} \mathrm{HCl}$ solutions in the absence and presence of inhibitor at various concentrations are shown in Figure 2. The Nyquist plots obtained are semicircles. The simple -R(CR)- model describes best the situation at the metal solution interface. However, the complex plane plots obtained are depressed semicircles, indicating non-ideal capacitive behavior of metal-solution interface. This capacitance dispersion at the solid surfaces can be attributed to the surface roughness, the degree of polycrystallinity, the chemical inhomogeneities and anion adsorption. A precise modeling for the experimental results was obtained by replacing a capacitor by a constant phase element (CPE) in the equivalent (Figure 3), whose impedance function is given as ${ }^{19}$ :

Table 1. Inhibition efficiency from weight loss measurement for mild steel in $1 \mathrm{M} \mathrm{HCl}$ solutions in the presence and absence of inhibitor.

\begin{tabular}{ccc}
\hline $\begin{array}{c}\text { Inhibitor } \\
\text { Concentration / ppm }\end{array}$ & $\begin{array}{c}\text { Corrosion } \\
\text { rate / mpy }\end{array}$ & $\begin{array}{c}\text { Inhibition } \\
\text { efficiency }(I E \%)\end{array}$ \\
\hline Blank & 168 & - \\
\hline 20 & 19.08 & 88.64 \\
\hline 40 & 17.19 & 89.77 \\
\hline 60 & 15.29 & 90.90 \\
\hline 100 & 11.46 & 93.18 \\
\hline 200 & 7.64 & 95.45 \\
\hline
\end{tabular}

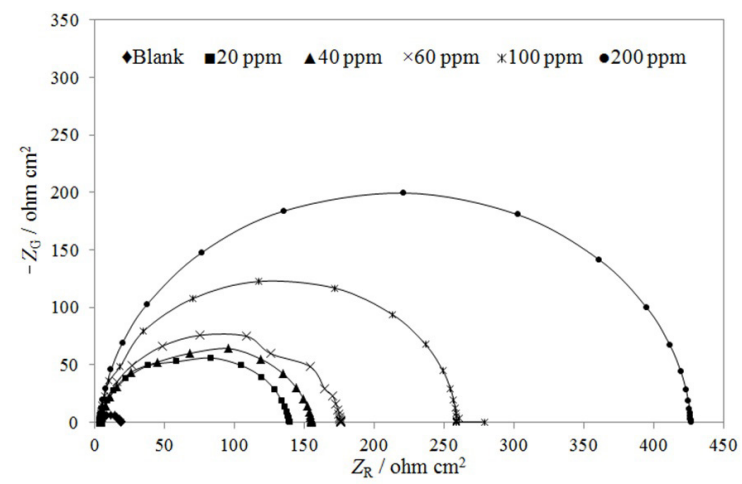

Figure 2. The Nyquist plots for MS electrode obtained in $1 \mathrm{M} \mathrm{HCl}$ solutions in the absence and presence of (2,2'-(2-Hydroxyethylimino) bis[N-(alphaalpha-dimethylphenethyl)-N-methyl acetamide] at various concentrations. 


$$
Z_{C P E}=-\left(\frac{1}{Y_{0}(j \omega)^{n}}\right)
$$

where $Y_{0}$ represents the admittance of the corrosive system at $1 \mathrm{rad} \mathrm{s}^{-1}$ and $n$, a constant $(-1 \leq n \leq 1)$. When $n=0, \mathrm{CPE}$ represents a pure resistor, if $n=+1$ the CPE represents a pure capacitor and an inductor if $n=-1$.

The electrochemical impedance parameters obtained by fitting the experimental curves into an equivalent circuit shown in Figure 3, are given in Table 2. In this equivalent circuit, polarization resistance $R$ indicates the contributions of charge transfer resistance, film resistance, accumulation resistance and double layer resistance. The results demonstrate that the polarization resistance values increase and the double layer capacitance $\left(C_{\mathrm{dl}}\right)$ values decrease with an increase in inhibitor concentration. The increase in the $R_{\mathrm{p}}$ values with inhibitor concentration indicates an increase in the surface coverage by the inhibitor molecules, resulting in an increase in inhibitor efficiency ${ }^{44,45}$. The decrease in $C_{\mathrm{dl}}$ values is caused by decreasing in the surface area of the metal, which is in contact with corroding medium. The adsorption of inhibitor molecules on the metal surface takes place by replacing water molecules at the metal-solution interface that lead to decreasing in local dielectric constant and/or an increase in the electrical double layer. Hence, the change in $C_{\mathrm{dl}}$ values

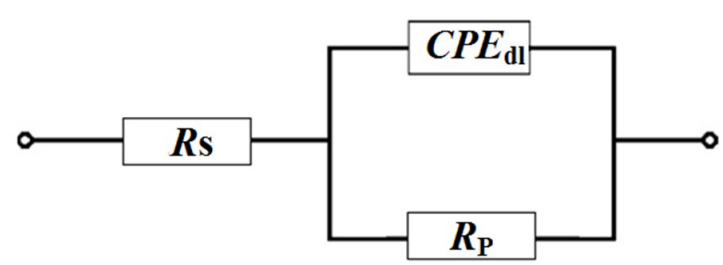

Figure 3. Equivalent circuit for the mild steel surface/corrosive media interface with CPE.

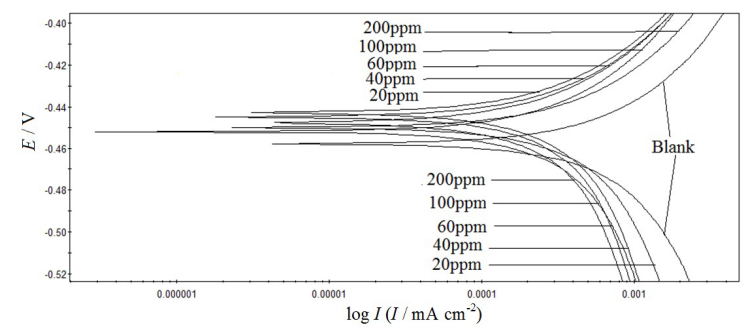

Figure 4. Tafel plots for mild steel in $1.0 \mathrm{M} \mathrm{HCl}$ solution in the absence and presence of the inhibitor at various concentrations. cause by the gradual displacement of water molecules by the adsorption of the inhibitor molecules at the metal-solution interface decreases the extent of metal corrosion ${ }^{46}$. From Table 2, it can be seen that the value of the constant ' $n$ ', which is a measure of the surface heterogeneity, increases with the inhibitor concentration towards unity, indicating that the mild steel surface becomes more and more homogeneous as the concentration of the inhibitor increases due to its uniform surface coverage at higher concentration.

\subsection{Potentiodynamic polarization studies}

The potentiodynamic polarization curves recorded for the corrosion of mild steel in $1 \mathrm{M} \mathrm{HCl}$ solutions in the presence and absence of inhibitor at various concentrations are shown in Figure 4. From this figure it is clear that the addition of inhibitor to the corrosive medium shows a reduction of both anodic and cathodic currents. This indicates that the inhibitor is of mixed type. Moreover, the cathodic branches in the presence of inhibitor give rise to almost parallel lines, indicating that the increase in inhibitor concentration doesn't modify the hydrogen evolution mechanism and the reduction of $\mathrm{H}^{+}$ions at the mild steel surface is charge transfer controlled ${ }^{47,48}$. The corresponding electrochemical parameters obtained from polarization studies (Tafel plots) are shown in Table 3. As it is apparent from Table 3, the corrosion current density values decrease with an increase in inhibitor concentration. The values of Tafel constants $\left(\beta_{\mathrm{c}}\right.$ and $\left.\beta_{\mathrm{a}}\right)$ also change with inhibitor concentration indicating that the addition of inhibitor affects both cathodic and anodic reactions. However, a higher $\beta_{\mathrm{c}}$ value than $\beta_{\mathrm{a}}$ in the presence of inhibitor indicates that the cathodic reaction is retarded to a higher extent than anodic reaction. This behavior can be also seen from the Tafel plots, where the cathodic curves are shifted toward lower current densities to a higher extent than the anodic curves with an increase in inhibitor concentration. The $E_{\text {corr }}$ values are also shifted to more negative side with an increase in inhibitor concentration. All these could be attributed to the decrease in the rate of hydrogen evolution reaction caused by the adsorption of inhibitor molecules on the mild steel surface ${ }^{49}$.

According to Tables 1 and 3, the variation of calculated corrosion rates with respect to inhibitor concentrations from weight loss and Tafel polarization have the same manner but with almost different values. This is due to high applied cathodic and anodic overpotentials in the Tafel diagrams. More accurate data can be obtained by weight loss method. Moreover, weight loss is according to standard for corrosion rate calculation but needs long time. For weight loss method, test condition is more similar to natural situation and therefore more precision data can be calculated. The predominance

Table 2. Impedance parameter values for the corrosion of mild steel in $1 \mathrm{~N} \mathrm{HCl}$ and $1 \mathrm{~N} \mathrm{H}_{2} \mathrm{SO}_{4}$ solutions in the presence and absence of inhibitor.

\begin{tabular}{|c|c|c|c|c|c|}
\hline Concentration / ppm & $R_{\mathrm{p}} / \Omega \mathrm{cm}^{2}$ & $Y_{0}\left(\times 10^{-6}\right) / \Omega^{-1} \mathrm{~cm}^{-2}$ & $n$ & $C_{\mathrm{dl}} / \mu \mathrm{Fcm}^{-2}$ & $I E \%$ \\
\hline Blank & 14.8 & 304 & 0.902 & 116.5 & - \\
\hline 20 & 135.8 & 92.4 & 0.911 & 38.2 & 89.1 \\
\hline 40 & 151.1 & 78.5 & 0.913 & 31.2 & 90.2 \\
\hline 60 & 172.1 & 64.3 & 0.929 & 25.4 & 91.4 \\
\hline 100 & 255.2 & 31.4 & 0.978 & 19.7 & 94.2 \\
\hline 200 & 422.9 & 27.7 & 0.963 & 10.4 & 96.5 \\
\hline
\end{tabular}


Table 3. Tafel polarization curves for mild steel in $1 \mathrm{~N} \mathrm{HCl}$ and $1 \mathrm{~N} \mathrm{H}_{2} \mathrm{SO}_{4}$ solutions in the presence and absence of inhibitor.

\begin{tabular}{ccccccc}
\hline Concentration $/ \mathrm{ppm}$ & $E_{\text {corr }} / \mathrm{mV}$ & $I_{\text {corr }} / \mu \mathrm{A} \mathrm{cm}{ }^{-2}$ & $\beta_{\mathrm{c}} / \mathrm{mV}$ & $\beta_{\mathrm{a}} / \mathrm{mV}$ & $\begin{array}{c}\text { Corrosion } \\
\text { rate } / \mathrm{mpy}\end{array}$ & $I E \%$ \\
\hline Blank & -429 & $1.88 \times 10^{3}$ & 127 & 68 & 858.27 & - \\
\hline 20 & -448 & $2.09 \times 10^{2}$ & 228 & 103 & 95.41 & 89 \\
\hline 40 & -451 & $1.88 \times 10^{2}$ & 219 & 109 & 85.83 & 90 \\
\hline 60 & -452 & $1.69 \times 10^{2}$ & 286 & 140 & 77.15 & 91 \\
\hline 100 & -452 & $1.32 \times 10^{2}$ & 292 & 179 & 60.26 & 93 \\
\hline 200 & -454 & $7.50 \times 10^{1}$ & 331 & 151 & 34.24 & 96 \\
\hline
\end{tabular}

of potentiodynamic polarization technique which used in most of research is the corrosion rate calculation in very low experiment time. In addition, the obtained inhibition efficiencies by Tafel are in agreement with weight loss and impedance measurements.

\subsection{Adsorption isotherm}

Adsorption isotherm provides information about the nature of interaction between inhibitor molecules and metal surface ${ }^{50}$. As mentioned earlier, the adsorption process can be considered as the replacement of water molecules at the corroding surface by inhibitor molecules ${ }^{51}$. Various adsorption isotherms were tested for the data obtained in weight loss measurements (also impedance and Tafel) and the data fit well with the Langmuir Adsorption isotherm, given by the expression;

$$
\frac{C_{i n h}}{\theta}=C_{i n h}+\frac{1}{K_{a d s}}
$$

Where $C_{\text {inh }}$ is concentration of the inhibitor, $\theta$ is fraction of total surface covered by inhibitor molecules and $K_{\text {ads }}$ is adsorption equilibrium constant. The plot is linear with a correlation coefficients higher than 0.99 . The value of $K_{\text {ads }}$, determined from the plot of $C_{\text {inh }} / \theta$ vs. $C_{\text {inh }}$ at constant temperature is used to calculate the value of standard free energy of adsorption $\left(\Delta G_{\text {ads }}\right)$ using the expression ${ }^{52}$ :

$$
K_{a d s}=1 / 55.5 \exp ^{(-\Delta G / R T)}
$$

The calculated value of $\Delta G_{\text {ads }}$ for adsorption of inhibitor molecules on the mild steel surface in $1 \mathrm{M} \mathrm{HCl}$ solution is $-32 \mathrm{~kJ} \mathrm{~mol}^{-1}$. The negative value of $\Delta G_{\text {ads }}$ shows that the interaction between the inhibitor molecule and metal surface is strong and the adsorption of the inhibitor molecules on to the mild steel surface takes place spontaneously ${ }^{53,54}$. In general, the values of $-\Delta G_{\text {ads }}$ less than $20 \mathrm{~kJ} \mathrm{~mol}^{-1}$ imply the columbic electrostatic interaction between the charged molecules and the metal surface ${ }^{55,56}$ and greater than $40 \mathrm{~kJ} \mathrm{~mol}^{-1}$ imply the formation of chemical bond between the inhibitor molecules and the atoms on the metal surface through charge sharing or charge transfer ${ }^{57}$. Any value in between these two values corresponds to adsorption of inhibitor molecules on the metal surface by both physisorption and chemisorption. In the present study the calculated $-\Delta G_{\text {ads }}$ value is close to $40 \mathrm{~kJ} \mathrm{~mol}^{-1}$ and far from $20 \mathrm{~kJ} \mathrm{~mol}^{-1}$, indicating that the adsorption of inhibitor molecules on the metal surface involves both physisorption and chemisorption.

\subsection{Effect of temperature}

The change of corrosion rate with the temperature was studied in $\mathrm{M} \mathrm{HCl}$ solutions with and without inhibitor by weight loss measurements at the temperatures 30,40 and $50{ }^{\circ} \mathrm{C}$

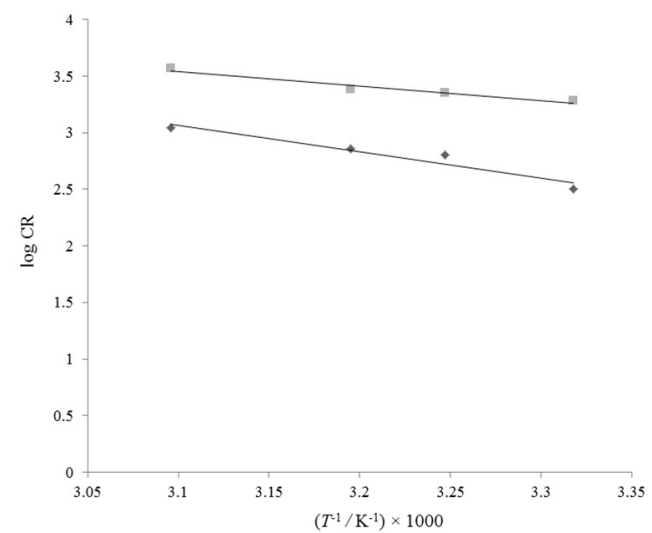

Figure 5. Typical Arrhenius plots for corrosion of mild steel in $1 \mathrm{M} \mathrm{HCl}$ solutions in the absence ( $\square$ ) and presence $(\downarrow)$ of inhibitor $(200 \mathrm{mg} / \mathrm{L})$ at various temperatures.

by weight loss measurements. The variation of corrosion rate with temperature is shown in Figure 5 using the Arrhenius type plot according to the equation ${ }^{58}$ :

$$
\ln (\text { corrosion rate })=\ln A-E_{a} / R T
$$

The calculated values of activation energy from the slopes of Arrhenius type plots are $72.34 \mathrm{~kJ} \mathrm{~mol}^{-1}$ and $64.23 \mathrm{~kJ}$ $\mathrm{mol}^{-1}$ for the uninhibited and inhibited (200 ppm) solutions respectively. The lower activation energy in the presence of inhibitor suggests that the inhibitor molecules are adsorbed on the metal surface by chemisorptions ${ }^{59}$.

\subsection{Potential of zero charge (PZC) and inhibition mechanism}

The adsorption of inhibitor molecules on the metal surface generally depends on the surface charge of the metal, the charge on the inhibitor molecule or the dipole moment of the inhibitor molecule and the other ions that are specifically adsorbed on the metal surface ${ }^{53}$. The surface charge on the metal surface in the corroding medium is determined from the position of open circuit potential with respect to $\mathrm{PZC}^{54}$. At the open circuit potential the surface charge on the mild steel surface is given by the expression:

$$
E_{r}=E_{O C P}-E_{P Z C}
$$

where $E_{\mathrm{r}}$ is Antropov's "rational" corrosion potential ${ }^{60}$. The values of $E_{\mathrm{OCP}}$ and $E_{\mathrm{r}}$ determined from Figure 6 for mild steel in $1 \mathrm{M} \mathrm{HCl}$ solutions in the presence and absence of inhibitor are given in Table 4. As it is clear from this table that the surface charge on the mild steel surface in 
the inhibited and uninhibited solutions to be positive. Being Lewis base, in the acidic medium some of the inhibitor molecules can protonate using the lone pair of electrons on the heteroatoms $(\mathrm{O}$ and $\mathrm{N})$ and the protonated molecules will have positive charge. Based on the surface charge and the positive charge on the protonated inhibitor molecules the following mechanism was proposed for the corrosion of mild steel in $1 \mathrm{M} \mathrm{HCl}$ solutions in the presence and absence of inhibitor ${ }^{54,60,61}$. In the uninhibited $\mathrm{HCl}$ solution, at the open circuit potential the metal surface is positively charged, the dissolution of metal involves successively the reversible adsorption of the $\mathrm{Cl}^{-}$ions to the mild steel surface, release of electrons from the adsorbed anions to the metal surface and desorption of the adsorbed anion along with $\mathrm{Fe}^{+2}$ ions, after picking up the electrons from the $\mathrm{Fe}$ atoms $^{60,61}$. In the inhibited $1 \mathrm{M} \mathrm{HCl}$ solution, the protonated

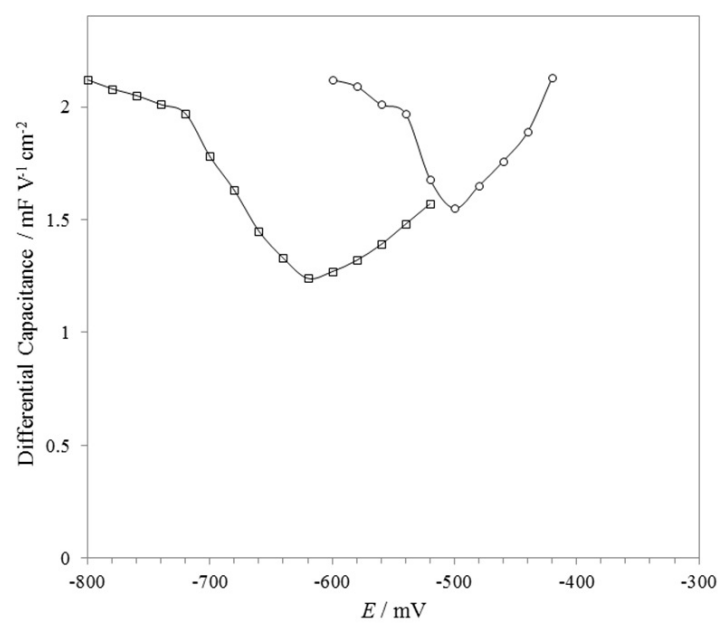

Figure 6. The plot of differential capacitance vs. applied electrode potential in $1 \mathrm{M} \mathrm{HCl}$ solution (o) and $1 \mathrm{M} \mathrm{HCl}$ containing $200 \mathrm{mg} / \mathrm{L}$ of the inhibitor solution $(\square)$.

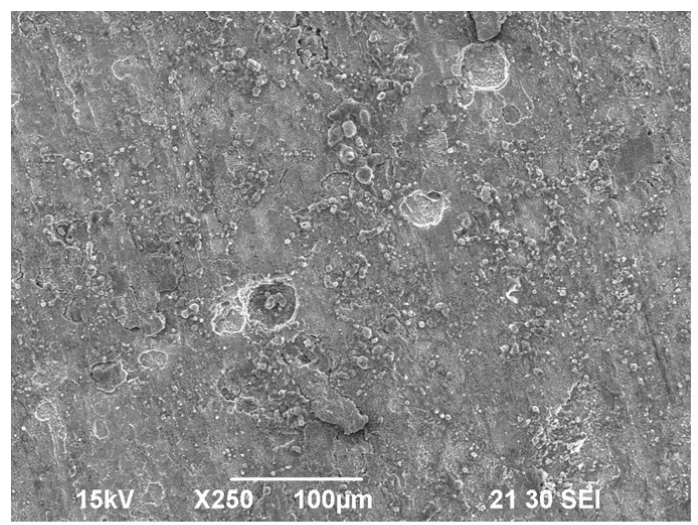

(a) inhibitor molecules at $\mathrm{O}$ and $\mathrm{N}$ atoms are in equilibrium with the corresponding molecular (unprotonated) form. Due to electrostatic repulsion, it is difficult for a positively charged molecule to approach the positively charged metal surface. The positively charged molecules can get adsorbed on the mild steel surface via $\mathrm{Cl}^{-}$ions, which form connecting bridge between the protonated inhibitor molecules and the metal surface $^{62,63}$. In addition to this type of physical adsorption, the adsorption of unprotonated form of inhibitor molecules can also take place through donor acceptor interactions between $\pi$ electrons of, benzene rings and the lone pair of electrons on $\mathrm{N}$ and $\mathrm{O}$ atoms and the vacant $\mathrm{d}^{\mathrm{d}}$ orbitals of surface iron atoms.

\subsection{Scanning electron microscopy}

Figure $7 \mathrm{a}$ and $\mathrm{b}$ show the SEM micrographs of the mild steel specimen immersed in the $1 \mathrm{M} \mathrm{HCl}$ solutions in the absence and presence of inhibitor respectively. The SEM monograph of mild steel specimen shows localized attack whereas the SEM monograph of mild steel specimen immersed in the inhibited solution indicates improvement in the surface morphology which could be due to the blocking of active centers on the mild steel surface by the inhibitor molecules.

\subsection{Molecular orbital theoretical studies}

Some of the molecular properties, describing the global reactivity such as; the total energy $\left(E_{T}\right)$, the energy of the Highest Occupied Molecular Orbital $\left(E_{\text {НОмO }}\right)$, the energy of the Lowest Unoccupied Molecular Orbital $\left(E_{L U M O}\right)$, the dipole moment, the Ionization Potential $(I)$, the Electron Affinity $(A)$, the electronegativity $(\chi)$, the global hardness $(\eta)$, the global softness $(S)$, the fraction of electron transferred $(\Delta \mathrm{N})$ and electrophilicity index $(\omega)$ are calculated using quantum chemical calculations. Other parameters describing

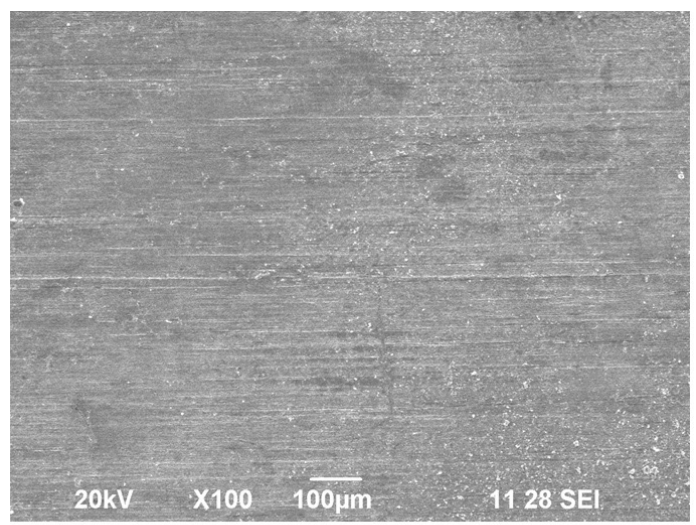

(b)

Figure 7. SEM monographs of mild steel specimen immersed in $1 \mathrm{M} \mathrm{HCl}$ solutions in the absence (a) and presence (b) of inhibitor.

Table 4. Excess charge on $\mathrm{MS}$ electrode in $1 \mathrm{~N} \mathrm{HCl}$ and $1 \mathrm{~N} \mathrm{H}_{2} \mathrm{SO}_{4}$ solutions in the presence and absence of inhibitor.

\begin{tabular}{lccc}
\hline \multicolumn{1}{c}{ Medium } & $\mathrm{E}_{\text {ocp }}(\mathrm{mV})$ & PZC $(\mathrm{mV})$ & Excess Charge \\
\hline $1 \mathrm{M} \mathrm{HCl}$ & -429 & -500 & + \\
\hline $1 \mathrm{M} \mathrm{HCl}+200 \mathrm{ppm}$ of inhibitor & -454 & -620 & + \\
\hline
\end{tabular}


the local selectivity of the studied molecule such as the local charge populations and the condensed Fukui functions are also considered. Some of these molecular parameters can be estimated following various approaches ${ }^{64}$. For instance, in the molecular orbital theory, the Koopmans' theorem ${ }^{64}$ stipulates that the HOMO energy is related to $I$ whereas the LUMO energy is linked to $A$, as follows:

$$
\begin{aligned}
& I=-E_{\text {Номо }} \\
& A=-E_{\text {LUMO }}
\end{aligned}
$$

According to Pearson, operational and approximate definitions of the electronic chemical potential $(\mu)$ and the absolute hardness $(\eta)$ of a chemical system are given by the following equations:

$$
\begin{gathered}
-\mu=\frac{(I+A)}{2}=\chi \\
\eta=\frac{(I-A)}{2}
\end{gathered}
$$

While the global softness, which is the inverse of the global hardness, is given as:

$$
S=\frac{1}{2 \eta}
$$

The fraction of transferred electrons $(\Delta \mathrm{N})$, evaluating the electronic flow in a reaction of two systems with different electronegativities, in particular case; a metallic surface and an inhibitor molecule, is calculated according to Pearson theory as follows ${ }^{65}$ :

$$
\Delta N=\frac{\chi_{M}-\chi_{I}}{2\left(\eta_{M}+\eta_{I}\right)}
$$

where the subscripts $M$ and $I$ represent the metal and inhibitor, respectively. $\eta_{M}$ and $\eta_{I}$ denote the absolute hardness of $\mathrm{Fe}$ atom and the inhibitor molecule, respectively. A theoretical value for the electronegativity of bulk iron is $\chi_{M}=7 \mathrm{eV}$ and a global hardness is $\eta_{M}=0 \mathrm{eV}$, by assuming that for a metallic bulk $I=A$, because they are softer than the neutral metallic atoms. Recently, Li and Parr ${ }^{66}$ and Gomez et al ${ }^{67}$ and Chattaraj et al. ${ }^{68}$ and $\mathrm{Liu}^{69}$ have introduced an electrophilicity index $(\omega)$ defined as;

$$
\omega=\frac{\mu^{2}}{2 \eta}
$$

Recent progress in computational methods has provided a very useful tool for understanding the properties and for describing the behavior of atoms in molecules. Recently, computational methods have become very popular due to their accuracy and less computational burden. The mechanism of the inhibition action can be elucidated with the help of quantum chemical calculation, which is widely reported in the literature ${ }^{70-72}$. In this study, quantum chemical calculations are carried out at two different methods; $a b$ initio methods at the Hartree-Fock (HF) level and the density functional theory (DFT) level with the $3-21 \mathrm{G}$ and $6-31 \mathrm{G}(\mathrm{d}, \mathrm{p})$ basis sets by geometry optimization of the studied molecule to establish the relationship between the molecular structure of 2,2'-(2-Hydroxyethylimino) bis[N-(alphaalpha-dimethylphenethyl)-N-methylacetamide] and its inhibition effects. It is obvious that the inhibition performance of an inhibitor on a metal surface is determined by the interplay of both spatial molecular and electronic parameters $^{71}$. Figure 8 represents the optimized structure of 2,2'-(2-Hydroxyethylimino)bis[N-(alphaalphadimethylphenethyl)-N-methylacetamide]. The selected bond lengths and bond angles for 2,2'-(2-Hydroxyethylimino) bis[N-(alphaalpha-dimethylphenethyl)-N-methylacetamide] are tabulated in Table 5. It can be seen that, the studied molecule is not completely planar, which may result in relatively weak interaction between molecule and metal surface. However, elucidation of orientation of organic molecules on the electrode surface needs to consider different factors. The interaction between atoms and groups of the inhibitor molecules and the electrode surface depend on the geometry of the inhibitor as well as the nature of their frontier molecular orbitals.

The HOMO and LUMO populations of the studied molecule are shown in Figure 9. It can be seen in Figure 9 that, the frontier molecular orbital distribution obtained from four different calculation methods have given very close results. The compound investigated in the present study contains of two symmetrical parts consisting of two benzene rings and two imide groups. The LUMO location of inhibitor is relatively

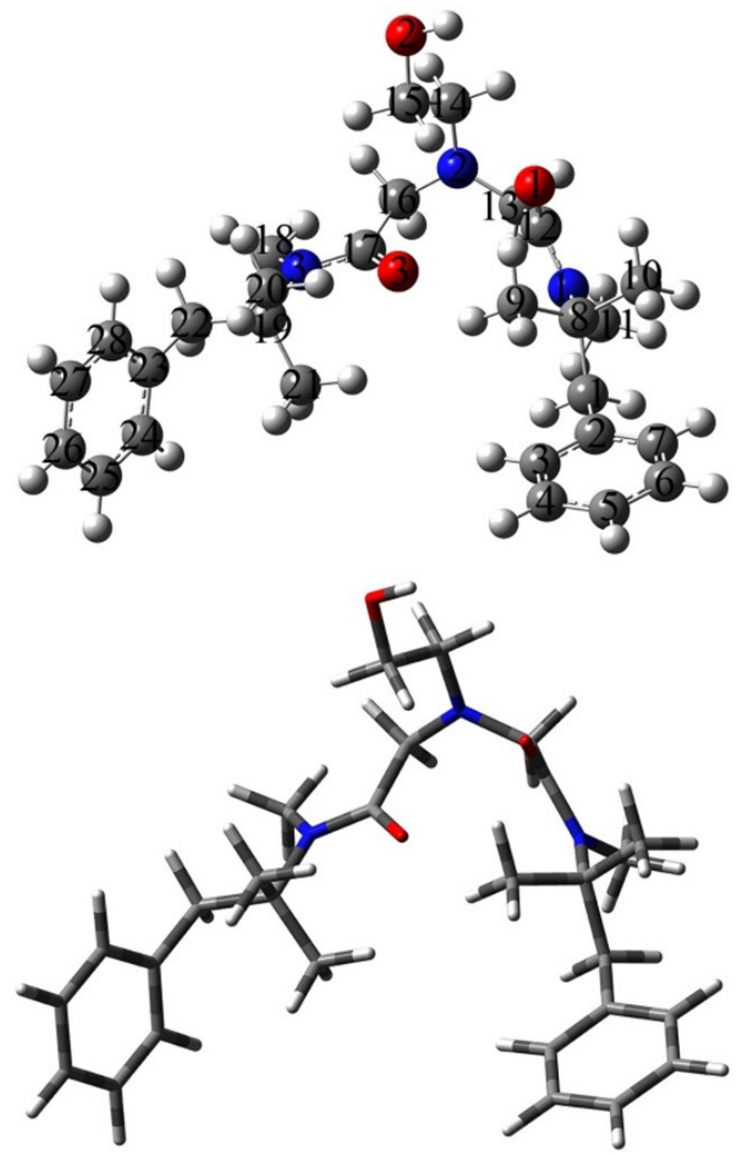

Figure 8. Different perspective of DFT optimized structure of 2,2'-(2-Hydroxyethylimino)bis[N-(alphaalpha-dimethylphenethyl)N-methylacetamide] obtained at the B3LYP/6-31G(d,p). 
Table 5. Selected Bond Lengths (in angstroms) and Bond Angles (in degrees) of the oxetacaine with B3LYP/6-31G(d,p).

\begin{tabular}{|c|c|}
\hline & Oxetacaine \\
\hline \multicolumn{2}{|c|}{ Bong lengths } \\
\hline $\mathrm{C} 1-\mathrm{C} 2$ & 1.51891 \\
\hline $\mathrm{C} 1-\mathrm{C} 8$ & 1.57368 \\
\hline C8-C9 & 1.54404 \\
\hline $\mathrm{C} 8-\mathrm{C} 10$ & 1.54313 \\
\hline C8-N1 & 1.52506 \\
\hline $\mathrm{N} 1-\mathrm{C} 12$ & 1.37926 \\
\hline N1-C11 & 1.47386 \\
\hline $\mathrm{C} 12-\mathrm{O} 1$ & 1.25651 \\
\hline $\mathrm{C} 12-\mathrm{C} 13$ & 1.54886 \\
\hline C13-N2 & 1.45299 \\
\hline N2-C14 & 1.46445 \\
\hline $\mathrm{C} 14-\mathrm{C} 15$ & 1.53759 \\
\hline $\mathrm{C} 15-\mathrm{O} 2$ & 1.46285 \\
\hline N2-C16 & 1.44272 \\
\hline $\mathrm{C} 16-\mathrm{C} 17$ & 1.55497 \\
\hline $\mathrm{C} 17-\mathrm{O} 3$ & 1.25633 \\
\hline C17-N3 & 1.38034 \\
\hline N3-C18 & 1.47461 \\
\hline N3-C19 & 1.53139 \\
\hline $\mathrm{C} 19-\mathrm{C} 20$ & 1.54441 \\
\hline $\mathrm{C} 19-\mathrm{C} 21$ & 1.54391 \\
\hline $\mathrm{C} 19-\mathrm{C} 22$ & 1.57095 \\
\hline $\mathrm{C} 22-\mathrm{C} 23$ & 1.51985 \\
\hline Avg. of $(\mathrm{C}-\mathrm{C})_{\mathrm{Ar}}$ & 1.40184 \\
\hline \multicolumn{2}{|c|}{ Bond angles } \\
\hline C9-C8-C10 & 110.95813 \\
\hline C20-C19-C21 & 111.28902 \\
\hline C1-C8-N1 & 107.38026 \\
\hline C22-C19-N3 & 108.53638 \\
\hline N1-C12-C13 & 118.77695 \\
\hline N3-C17-C16 & 118.79765 \\
\hline C13-N2-C16 & 119.63392 \\
\hline C1-C8-N1-C12 & 161.48218 \\
\hline C8-N1-C12-C13 & -168.54489 \\
\hline N1-C12-C13-N2 & 139.89526 \\
\hline C12-C13-N2-C16 & -108.15446 \\
\hline C13-N2-C16-C17 & 76.44796 \\
\hline N2-C16-C17-N3 & 164.04930 \\
\hline C16-C17-N3-C19 & -176.78487 \\
\hline C17-N3-C19-C22 & 172.19852 \\
\hline $\mathrm{N} 2-\mathrm{C} 14-\mathrm{C} 15-\mathrm{O} 2$ & -179.98150 \\
\hline
\end{tabular}

distributed over the benzene ring and a small part of the carbon chain that occur one side of the molecule whereas the HOMO is entirely localized on the heteroatoms. According to HOMO distribution, it can be said that 2,2'-(2-Hydroxyethylimino) bis[N-(alphaalpha-dimethylphenethyl)-N-methylacetamide] molecule carries its rich negative center in a small region. The molecules are oriented toward to cathodic sites of the steel surface through the parts of the molecules with low HOMO density and afterwards adsorption occurs by sharing of electrons. In addition, the inhibitor molecule can accept electrons from a Fe atom with its antibonding orbitals to form back-donating. These donation and back-donation processes strengthen the adsorption of inhibitor onto the mild steel surface ${ }^{73}$.

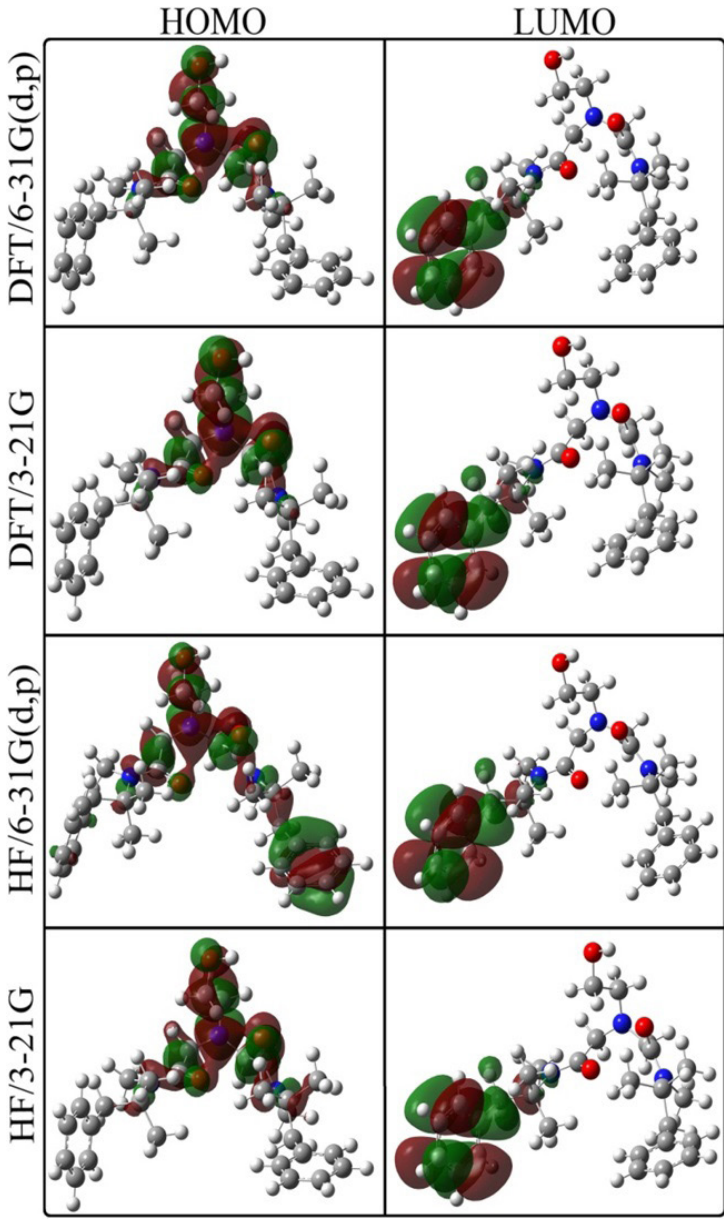

Figure 9. HOMO and LUMO populations of 2,2'-(2-Hydroxyethylimino) bis[N-(alphaalpha-dimethylphenethyl)-N-methylacetamide] obtained at different levels.

It is important to consider the situation corresponding to a molecule that is going to receive a certain amount of charge at some center and is going to back-donate a certain amount of charge through the same center or another ${ }^{74}$. To describe the energy change associated with these two processes, the second order simple charge transfer formula is regarded as a two-parameter expression, in which the donation and back-donation processes are differentiated through the use of the values of the chemical potential for each case, while the hardness is fixed to the value of $\eta=\left(\mu^{+}-\mu^{-}\right)$in both situations. Thus, when the molecule receives a certain amount of charge, $\Delta \mathrm{N}^{+74,75}$,

$$
\Delta E^{+}=\mu^{+} \Delta N^{+}+\frac{1}{2} \eta\left(\Delta N^{+}\right)^{2}
$$

and when the molecule back-donates a certain amount of charge, $\Delta \mathrm{N}^{-}$, then

$$
\Delta E^{-}=\mu^{-} \Delta N^{-}+\frac{1}{2} \eta\left(\Delta N^{-}\right)^{2}
$$

If the total energy change is approximated by the sum of the contributions from Equations 15 to 17, and the amount of charge back-donated is equal to the amount of charge received, $\Delta \mathrm{N}^{-}=-\Delta N^{+}$, then; 
Table 6. Some quantum chemical parameters for oxetacaine obtained at (A) B3LYP/6-31G(d,p), (B) B3LYP/3-21G, (C) HF/6-31G(d,p) and (D) HF/3-21G.

\begin{tabular}{lrrrr}
\hline \multicolumn{1}{c}{ quantum chemical properties } & $\mathrm{A}$ & $\mathrm{B}$ & $\mathrm{C}$ & $\mathrm{D}$ \\
\hline total energy $(\mathrm{eV})$ & -40305.741 & -40096.458 & -40041.844 & -39836.299 \\
\hline$E_{\text {LUMO }}(\mathrm{eV})$ & -0.116 & -0.077 & 3.831 & 3.885 \\
\hline$E_{\text {HOMO }}(\mathrm{eV})$ & -5.009 & -4.797 & -8.693 & -8.530 \\
\hline$\Delta E(\mathrm{eV})$ & 4.893 & 4.720 & 12.523 & 12.415 \\
\hline dipole moment $(\mathrm{D})$ & 5.827 & 5.729 & 5.259 & 5.881 \\
\hline ionization potential $(\mathrm{I} / \mathrm{eV})$ & 5.009 & 4.797 & 8.693 & 8.530 \\
\hline electron affinity $(A / \mathrm{eV})$ & 0.116 & 0.077 & -3.831 & -3.885 \\
\hline hardness $(\eta / \mathrm{ev})$ & 2.446 & 2.360 & 6.262 & 6.207 \\
\hline softness $(S / \mathrm{eV})$ & 0.204 & 0.212 & 0.080 & 0.081 \\
\hline electronegativity $(\chi)$ & 2.563 & 2.437 & 2.431 & 2.322 \\
\hline fraction of electrons transferred $(\Delta N)$ & 0.907 & 0.967 & 0.365 & 0.377 \\
\hline electrophilicity $(\omega)$ & 1.342 & 1.258 & 0.472 & 0.434 \\
\hline$\Delta E_{T}$ & -0.612 & -0.590 & -1.565 & -1.552 \\
\hline molar volume $\left(\mathrm{cm}^{3}\right.$ mol $\left.^{-1}\right)$ & 316.023 & 388.615 & 353.746 & 397.661 \\
\hline
\end{tabular}

$$
\Delta E_{T}=\Delta E^{+}+\Delta E^{-}=\left(\mu^{+}-\mu^{-}\right) \Delta N^{+}+\eta\left(\Delta N^{+}\right)^{2}
$$

The most favorable situation corresponds to the case when the total energy change becomes a minimum with respect to $\Delta \mathrm{N}^{+}$, which implies that $\Delta N^{+}=-\left(\mu^{+}-\mu^{-}\right) / 2 \eta$ and

$$
\Delta E_{T}=\frac{-\left(\mu^{+}-\mu^{-}\right)^{2}}{4 \eta}=-\frac{\eta}{4}
$$

The calculations from Table 6 indicate that $\eta>0$ and $\Delta \mathrm{E}_{T}<0$ in all used computational methods. This result implies that the charge transfer to inhibitor molecule followed by back-donation from the molecule is energetically favorable. Similar observation has been reported ${ }^{76}$. However, it is important to note that $\Delta \mathrm{E}_{T}$ values obtained don't predict that a back-donation process is going to occur; it only establishes that if both processes occur (charge transfer to the molecule and back-donation from the molecule), the energy change is directly proportional to the hardness of the molecule.

The binding ability of the inhibitor to the metal surface increases with increasing HOMO and decreasing LUMO energy values. Thus, the lower the value of $E_{L U M O}$, the most probable it is that the molecule would accept electrons. Moreover, the gap between the HOMO and LUMO energy levels $(\Delta \mathrm{E})$ of the molecule is an important parameter that determines the reactivity of the inhibitor molecule toward the adsorption on the metallic surface. In addition to $\Delta \mathrm{E}$ parameter, for effective overlapping, the energy difference between the orbitals generally must be low, and the overall energy difference between the orbitals (HOMO and HOMO-1) is $1.02 \mathrm{eV}$ for the studied compound, indicating their participation in the metal-ligand interaction. In addition, the energy difference between the HOMO-2 and HOMO- 1 is very low confirming the involvement of HOMO- 1 and HOMO-2 in the ligand-metal interaction (as shown in Figure 10) and also, suggesting that the parameters of orbitals such as HOMO, HOMO-1 and HOMO-2 are important for chemical reactivity over metal surface ${ }^{77-79}$.

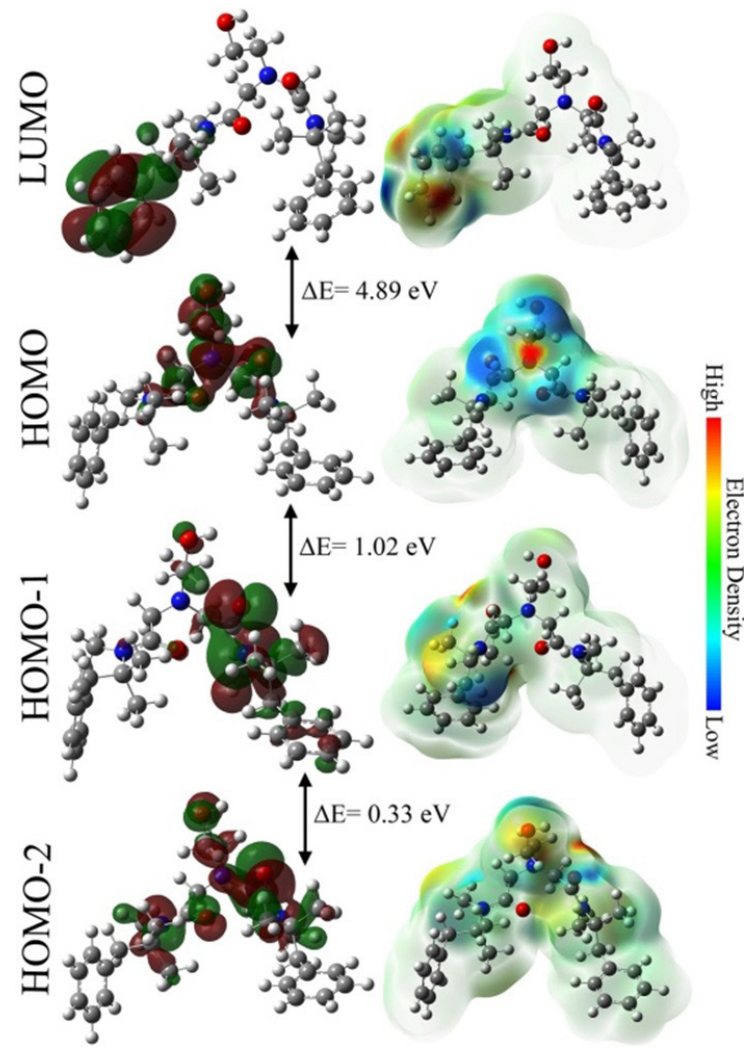

Figure 10. (Left) molecular orbitals LUMO, HOMO, HOMO-1 and HOMO-2 of 2,2'-(2-Hydroxyethylimino)bis[N-(alphaalphadimethylphenethyl)-N-methylacetamide] obtained at the B3LYP/6$31 \mathrm{G}(\mathrm{d}, \mathrm{p})$ level and their energies. (Right) the electron density of corresponding orbitals for the studied compound.

The fraction of electrons transferred from inhibitor to the iron molecule by different computational methods, is calculated and listed in Table 6 . According to other reports ${ }^{80,81}$, the values of $\Delta N$ show inhibition effect resulted from electrons donation. 
Agreeing with Lukovits's study ${ }^{81}$ if $\Delta N<3.6$, the inhibition efficiency increases with increasing electron-donating ability at the metal surface. Thus, in the present study, inhibitor molecule is donor of electrons and the mild steel surface is the acceptor of electrons. The electrophilicity index, $\omega$, which measures the electrophilic power of a molecule, is calculated for this molecule. The positive value of $\omega$ suggests the high capacity of the molecule to accept electrons ${ }^{82}$. This process increases the adsorption capacity of 2,2'-(2-Hydroxyethylimino) bis[N-(alphaalpha-dimethylphenethyl)-N-methylacetamide] on the steel surface. In a corroding system, it is important to note that the inhibitor acts as a Lewis base while the metal acts as a Lewis acid.

The Fukui functions indicate the regions on the inhibitor molecule on which nucleophilic and electrophilic reactions are likely to occur. The Fukui function is defined as ${ }^{83}$ :

$$
f(r)=\left[\frac{\partial^{2} E}{\partial v(r) \partial N}\right]=\left[\frac{\partial}{\partial v(r)}\right]_{N}=\left[\frac{\partial \rho(r)}{\partial N}\right]_{V}
$$

where $v(r)$ is the external potential, $\rho(r)$ is total charge density, and $N$ is the total number of electrons. Due to discontinuity in the $\rho(\mathrm{r})$ versus $N$ curve, we can define three different types of Fukui function, viz

$$
f_{k}^{+}=\left[q_{k}(N+l)-q_{k}(N)\right] \quad(\text { for nucleophilic attack) }
$$

$f_{k}^{-}=\left[q_{k}(N)-q_{k}(N-1)\right]$ (for electrophilic attack)

$f_{k}^{0}=\left[q_{k}(N+1)-q_{k}(N-1)\right] / 2$ (for radical attack)

Where $q_{k}(N), q_{k}(N+1)$ and $q_{k}(N-1)$ are the electron population of the $k^{T H}$ atom for $N, N+1$, and $N-1$ electron systems, respectively.

The preferred site for nucleophilic attack is the atom in the molecule where the value of $f^{+}$is the highest while the preferred site for electrophilic attack is the atom in the molecule where $f$ - has the highest value. However, the average of both measures reactivity towards a radical. The calculated values of the Fukui functions for the non-hydrogen atoms are reported in Table 7 . In addition to oxygen atoms, the value of $f^{+}$is highest on $\mathrm{C} 4, \mathrm{C} 7, \mathrm{C} 23$ and C26 atoms in 2,2'-(2-Hydroxyethylimino)bis[N-(alphaalphadimethylphenethyl)-N-methylacetamide] indicating that these atoms are likely to be the preferred sites for nucleophilic attack. These results agree with the analysis of the electron density of this molecule in the case of $N+1$ electron system which indicated that each $\mathrm{C}$ and $\mathrm{O}$ atoms is able to stabilize additional electrons (top panel of Figure 11). The value of $f$ -is highest on the same atoms approximately, in addition to N1 and N2 atoms. From bottom panel of Figure 11, it can be seen that these sites are the regions where electron removal destabilizes the molecule the least. The observed similarity in the sites for electrophilic and nucleophilic attacks suggests similarity in mechanism of inhibition.

The local reactivity of molecules is often analyzed in terms of the local softness index $s$, which is defined as the product of the Fukui function and the global softness, $S$. It is expressed using the equations ${ }^{84}$ :

$$
s^{+}=\left(f^{+}\right) S
$$

$$
\begin{aligned}
& s^{-}=\left(f^{-}\right) S \\
& s^{0}=\left(f^{0}\right) S
\end{aligned}
$$

Also, the relative nucleophilicity $\left(\mathrm{s}^{+} / \mathrm{s}^{-}\right)$and relative electrophilicity $\left(\mathrm{s}^{-} / \mathrm{s}^{+}\right)$are the indices that are also related to the local softness and can be applied for the prediction of the sites for nucleophilic and electrophilic attacks. It is observed that both the approaches and the local softness values give results similar to those obtained from the Fukui function calculations.

Figure 12 (top panel) shows the Mulliken atomic charges calculated for inhibitor compound. It has been reported that the more negative the atomic charges of the adsorbed center, the more easily the atom donates its electron to the unoccupied orbital of the metal ${ }^{85}$. It is clear from Figure 12, that the oxygen and nitrogen atoms as well as some carbon atoms carry negative charge centers which could offer electrons to the mild steel surface to form a coordinate bond. The direction of the dipole can be understood by considering the electrostatic potential (middle and bottom panels of Figure 12), which discerns electron density rich regions centered on the mentioned atoms, indicating the preferred zone for nucleophilic attack.

\subsection{Quantitative structure activity relationship (QSAR).}

An attempt has been made to correlate the results obtained from quantum chemical studies and the observed inhibition efficiencies of the inhibitor. The approach in which several

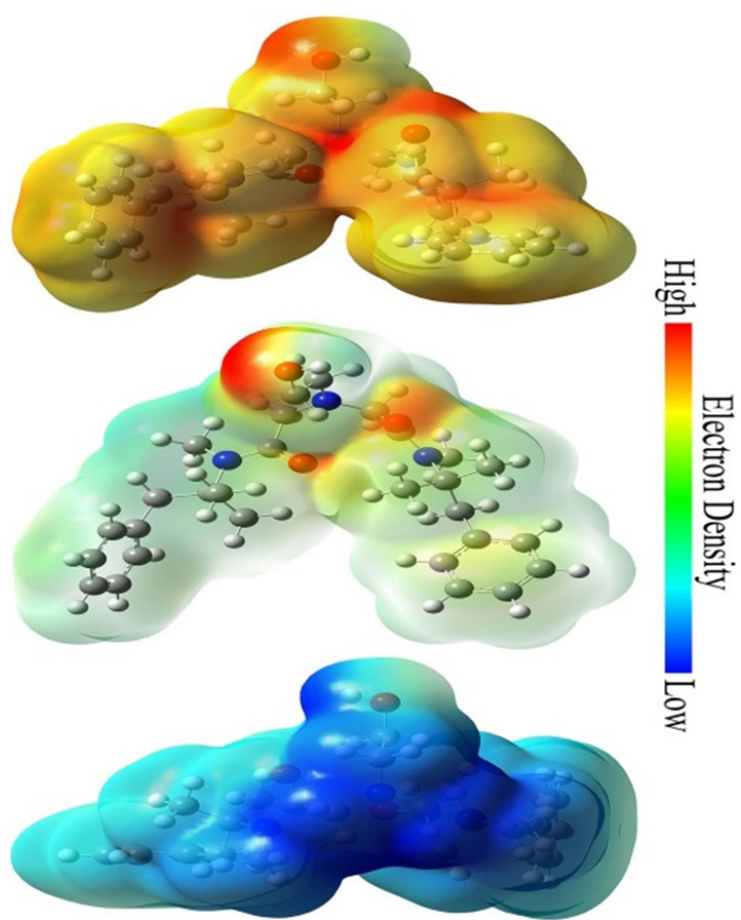

Figure 11. Iso-surface representation of electrostatic potential of 2,2'-(2-Hydroxyethylimino)bis[N-(alphaalpha-dimethylphenethyl)-Nmethylacetamide] as a system based on the independent single-point calculation for corresponding $N+1, N$ and $N-1$ electron systems, top to bottom respectively, at the B3LYP/6-31G(d,p) level. 
Table 7. The Mulliken population analysis of the condensed Fukui functions and local softness parameters on the selected atoms of oxetacaine.

\begin{tabular}{|c|c|c|c|c|c|c|c|c|c|}
\hline $\begin{array}{l}\text { Selected } \\
\text { atoms }\end{array}$ & $\begin{array}{l}\text { Mullikene } \\
\text { charge }\end{array}$ & $f_{k}^{+}$ & $f_{k}^{-}$ & $f_{k}^{o}$ & $S^{+}(e V)$ & $S^{-}(\mathrm{eV})$ & $S^{o}(e V)$ & $S^{+} / S^{-}$ & $S^{-} / S^{+}$ \\
\hline $\mathrm{C} 1$ & -0.328 & -0.001 & -0.005 & -0.003 & 0.000 & -0.001 & -0.001 & 0.290 & 3.445 \\
\hline $\mathrm{C} 2$ & 0.104 & -0.006 & -0.001 & -0.004 & -0.001 & 0.000 & -0.001 & 5.059 & 0.198 \\
\hline $\mathrm{C} 3$ & -0.151 & 0.019 & 0.001 & 0.010 & 0.004 & 0.000 & 0.002 & 23.742 & 0.042 \\
\hline $\mathrm{C} 4$ & -0.128 & 0.034 & 0.004 & 0.019 & 0.007 & 0.001 & 0.004 & 8.516 & 0.117 \\
\hline $\mathrm{C} 5$ & -0.122 & 0.001 & 0.007 & 0.004 & 0.000 & 0.001 & 0.001 & 0.076 & 13.238 \\
\hline C6 & -0.128 & 0.017 & 0.004 & 0.010 & 0.003 & 0.001 & 0.002 & 4.551 & 0.220 \\
\hline $\mathrm{C} 7$ & -0.152 & 0.035 & 0.001 & 0.018 & 0.007 & 0.000 & 0.004 & 26.153 & 0.038 \\
\hline $\mathrm{C} 8$ & 0.192 & -0.005 & -0.023 & -0.014 & -0.001 & -0.005 & -0.003 & 0.215 & 4.646 \\
\hline C9 & -0.425 & -0.002 & -0.003 & -0.002 & 0.000 & -0.001 & 0.000 & 0.810 & 1.235 \\
\hline $\mathrm{C} 10$ & -0.402 & -0.003 & 0.000 & -0.002 & -0.001 & 0.000 & 0.000 & 24.857 & 0.040 \\
\hline C11 & -0.249 & -0.009 & -0.016 & -0.013 & -0.002 & -0.003 & -0.003 & 0.533 & 1.877 \\
\hline $\mathrm{C} 12$ & 0.589 & 0.003 & 0.010 & 0.007 & 0.001 & 0.002 & 0.001 & 0.262 & 3.811 \\
\hline $\mathrm{C} 13$ & -0.147 & -0.004 & -0.050 & -0.027 & -0.001 & -0.010 & -0.006 & 0.076 & 13.073 \\
\hline $\mathrm{C} 14$ & -0.081 & -0.007 & -0.064 & -0.036 & -0.001 & -0.013 & -0.007 & 0.113 & 8.833 \\
\hline $\mathrm{C} 15$ & -0.040 & 0.002 & -0.007 & -0.002 & 0.000 & -0.001 & 0.000 & -0.326 & -3.070 \\
\hline $\mathrm{C} 16$ & -0.188 & -0.007 & -0.043 & -0.025 & -0.001 & -0.009 & -0.005 & 0.170 & 5.896 \\
\hline $\mathrm{C} 17$ & 0.553 & 0.017 & 0.012 & 0.015 & 0.004 & 0.002 & 0.003 & 1.445 & 0.692 \\
\hline $\mathrm{C} 18$ & -0.247 & -0.012 & -0.014 & -0.013 & -0.002 & -0.003 & -0.003 & 0.845 & 1.184 \\
\hline $\mathrm{C} 19$ & 0.196 & -0.011 & -0.017 & -0.014 & -0.002 & -0.003 & -0.003 & 0.642 & 1.558 \\
\hline $\mathrm{C} 20$ & -0.415 & -0.002 & -0.002 & -0.002 & 0.000 & 0.000 & 0.000 & 0.738 & 1.355 \\
\hline $\mathrm{C} 21$ & -0.405 & -0.004 & -0.001 & -0.002 & -0.001 & 0.000 & 0.000 & 6.457 & 0.155 \\
\hline $\mathrm{C} 22$ & -0.336 & -0.007 & -0.006 & -0.006 & -0.001 & -0.001 & -0.001 & 1.217 & 0.822 \\
\hline $\mathrm{C} 23$ & 0.104 & 0.023 & -0.002 & 0.011 & 0.005 & 0.000 & 0.002 & -13.928 & -0.072 \\
\hline $\mathrm{C} 24$ & -0.151 & 0.008 & 0.000 & 0.004 & 0.002 & 0.000 & 0.001 & 29.694 & 0.034 \\
\hline $\mathrm{C} 25$ & -0.128 & 0.014 & 0.003 & 0.008 & 0.003 & 0.001 & 0.002 & 4.832 & 0.207 \\
\hline $\mathrm{C} 26$ & -0.122 & 0.039 & 0.006 & 0.023 & 0.008 & 0.001 & 0.005 & 6.510 & 0.154 \\
\hline $\mathrm{C} 27$ & -0.128 & 0.002 & 0.003 & 0.003 & 0.000 & 0.001 & 0.001 & 0.808 & 1.237 \\
\hline $\mathrm{C} 28$ & -0.150 & 0.021 & 0.000 & 0.011 & 0.004 & 0.000 & 0.002 & 98.626 & 0.010 \\
\hline N1 & -0.546 & -0.003 & 0.012 & 0.005 & -0.001 & 0.003 & 0.001 & -0.201 & -4.969 \\
\hline N2 & -0.477 & -0.002 & 0.111 & 0.055 & 0.000 & 0.023 & 0.011 & -0.016 & -60.921 \\
\hline N3 & -0.553 & 0.000 & 0.008 & 0.004 & 0.000 & 0.002 & 0.001 & 0.031 & 32.623 \\
\hline $\mathrm{O} 1$ & -0.504 & 0.014 & 0.054 & 0.034 & 0.003 & 0.011 & 0.007 & 0.250 & 4.002 \\
\hline $\mathrm{O} 2$ & -0.619 & 0.010 & 0.056 & 0.033 & 0.002 & 0.012 & 0.007 & 0.169 & 5.915 \\
\hline O3 & -0.486 & 0.012 & 0.023 & 0.017 & 0.002 & 0.005 & 0.004 & 0.500 & 1.998 \\
\hline
\end{tabular}

quantum chemical parameters form a composite index that is correlated to the experimentally determined inhibition efficiency is called quantitative structure activity relationship. Several quantitative structure activity relationship equations are utilized to correlate the quantum chemical index with the experimental inhibition efficiencies. The selection of the appropriate model equation depends strongly on the type of adsorption mechanism. Among the equations utilized to develop quantitative structure activity relationship, when the adsorption of the inhibitor molecules on the metal surface obeys Langmuir adsorption isotherm, is the linear and the nonlinear multiple regression equations developed by
Lukovits $^{86,87}$. The linear and nonlinear multiple regression equations are given by the Equations 26 and 27 respectively;

$$
\begin{gathered}
I E_{\text {Theor }}=A x_{i} C_{i}+B \\
I E_{\text {Theor }}=\frac{\left(A x_{i}+B\right) C_{i}}{1+\left(A x_{i}+B\right) C_{i}} \times 100
\end{gathered}
$$

where $I E_{\text {Theor }}$ is the inhibition efficiency, $A$ and $B$ are the regression coefficients determined by regression analysis, $x_{i}$ is a characteristic quantum index for the inhibitor molecule $(i)$, and $C_{i}$ denotes the concentration of the inhibitor. Such linear 

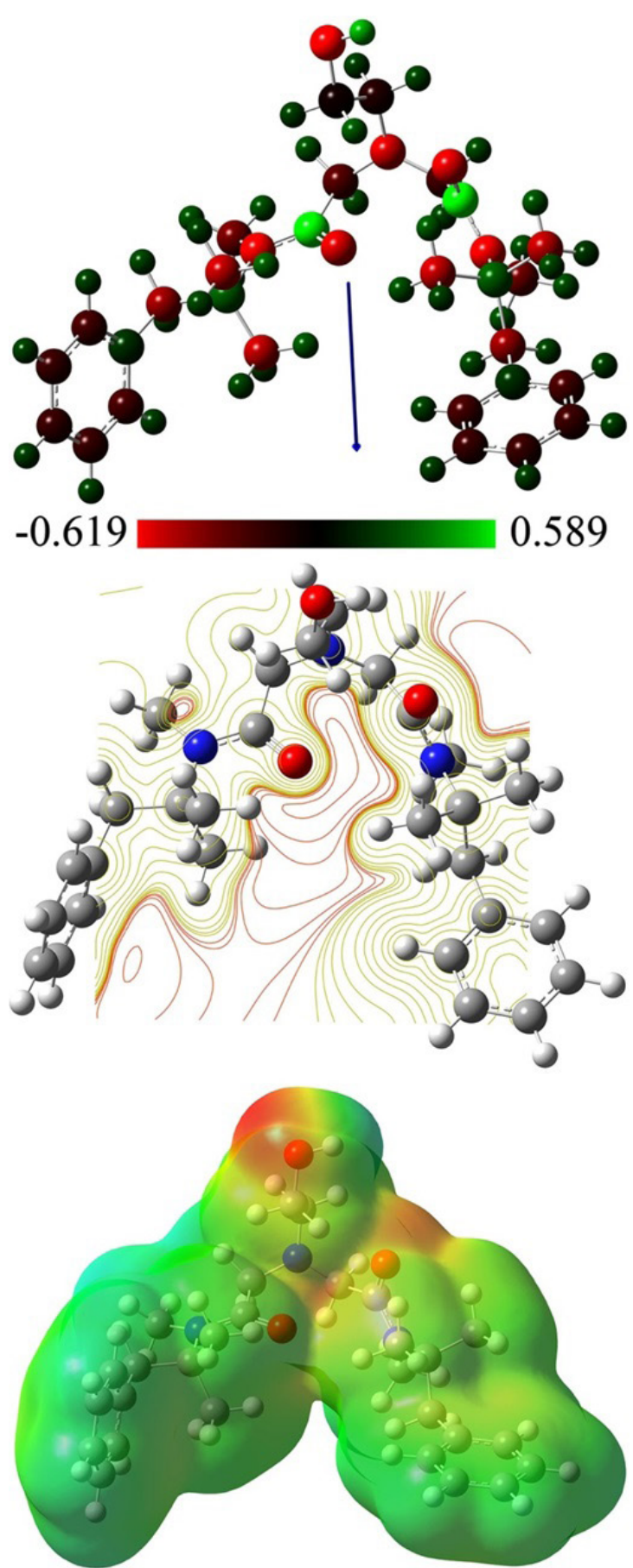

$-0.073$

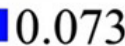

Figure 12. Electrostatic properties of 2,2'-(2-Hydroxyethylimino) bis[N-(alphaalpha-dimethylphenethyl)-N-methylacetamide] at the B3LYP/6-31G $(d, p)$ level: Mulliken charge population and the side view of the dipoles are displayed on the top while the middle and bottom panels show the contour and iso-surface representation of electrostatic potential, respectively.

approach is not found to be satisfactory for correlating the present results. Consequently, the nonlinear model (NLM) proposed by Lukovits et al. ${ }^{81}$ and also used by Khaled ${ }^{88}$ for studying the interaction of corrosion inhibitors with metal surfaces in acidic solutions based on the Langmuir adsorption isotherm and a combination of the quantum chemical parameters that provided the best correlation together with the Equations 28 to 31 were used for the prediction of theoretical inhibition efficiency at the B3LYP/3-21G and $\mathrm{HF} / 3-21 \mathrm{G}$ levels using the basis sets B3LYP/6-31G(d,p) and $\mathrm{HF} / 6-31 \mathrm{G}(\mathrm{d}, \mathrm{p})$ respectively.

$$
\begin{gathered}
\left(3.91 E_{\text {HOMO }}-8.22 E_{\text {LUMO }}+4.31 \Delta E+\right. \\
I E_{\text {Theor }}=\frac{2.06 \Delta N+4.51 \mu-0.10 \mathrm{~V}+1.45) C_{i}}{1+\left(3.91 E_{\text {HOMO }}-8.22 E_{\text {LUMO }}+4.31 \Delta E+\right.} \times 100 \\
2.06 \Delta N+4.51 \mu-0.10 \mathrm{~V}+1.45) C_{i}
\end{gathered}
$$

$$
I E_{\text {Theor }}=\frac{\begin{array}{l}
\left(1.05 E_{\text {HOMO }}+1.00 E_{\text {LUMO }}+0.95 \Delta E+\right. \\
1+\left(1.05 E_{\text {HOMO }}+1.00 E_{\text {LUMO }}+0.95 \Delta E+\right. \\
0.99 \Delta N+0.94 \mu-0.02 V+0.99) C_{i}
\end{array} \times 100}{2}
$$

$\left(1.06 E_{\text {HOMO }}+0.97 E_{\text {LUMO }}+0.92 \Delta E+\right.$

$$
\begin{aligned}
I E_{\text {Theor }}= & \frac{1.00 \Delta N+0.96 \mu-0.03 V+0.99) C_{i}}{1+\left(1.06 E_{\text {HOMO }}+0.97 E_{L U M O}+0.92 \Delta E+\right.} \times 100 \\
& 1.00 \Delta N+0.96 \mu-0.03 V+0.99) C_{i}
\end{aligned}
$$

$$
\begin{aligned}
& \left(1.06 E_{\text {HОMO }}+0.97 E_{L U M O}+0.91 \Delta E+\right. \\
& I E_{\text {Theor }}=\frac{1.00 \Delta \mathrm{N}+0.96 \mu-0.03 \mathrm{~V}+0.99) C_{i}}{1+\left(1.06 E_{\text {HOMO }}+0.97 E_{\text {LUMO }}+0.91 \Delta E+\right.} \times 100 \\
& 1.00 \Delta N+0.96 \mu-0.03 V+0.99) C_{i}
\end{aligned}
$$

From the results obtained, it can be seen that there is a good and acceptable coefficient correlation $\left(\mathrm{R}^{2}=0.8491\right)$ between the experimental and calculated/estimated inhibition efficiencies of the studlied inhibitor using the B3LYP/6-31G(d,p) method as shown in Figure 13A (other computational methods show the same results). Figure 13B shows a plot of the theoretical and experimental inhibition efficiencies versus the concentrations of the studied inhibitor, which confirms the accuracy of the obtained results from Figure 13A. It is also important to mention that it is difficult to predict the corrosion inhibition ability of a compound through an inclusive approach or to find some universal type of correlations. Other than a number of excluded parameters such as, effect of solvent molecules, surface nature, adsorption sites of the metal atoms or oxides sites or vacancies, competitive adsorption with other chemical species in the fluid phase and solubility should also be taken into consideration. Thus, the electron rich heteroatoms such as $\mathrm{O}$ and $\mathrm{N}$ can be protonated and this form could be in equilibrium with the molecular(unprotonated) form. The positively charged protonated form of 2,2'-(2-Hydroxyethylimino) bis[N-(alphaalpha-dimethylphenethyl)-N-methylacetamide] can get adsorbed on to the metal surface by physisorption. 

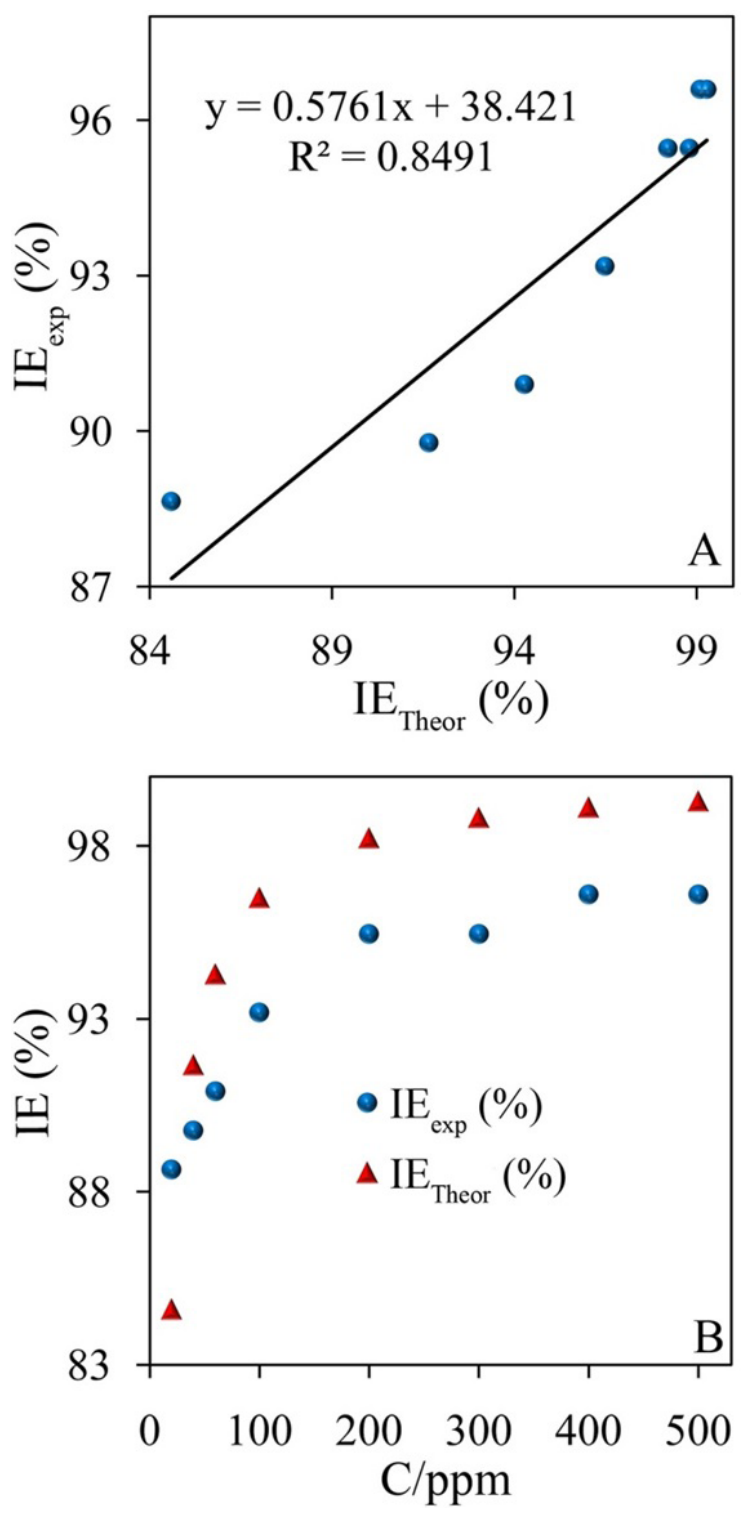

Figure 13. (A) Correlation of experimental and theoretical inhibition efficiencies of 2,2'-(2-Hydroxyethylimino) bis[N-(alphaalpha-dimethylphenethyl)-N-methylacetamide] using the B3LYP/6-31G(d,p) method. (B) Plot of $I E_{\text {exp }} \%$ and $I E_{\text {Theor }} \%$ versus 2,2'-(2-Hydroxyethylimino) bis [N-(alphaalphadimethylphenethyl)-N-methylacetamide] concentrations.

\section{Conclusion}

The adsorption and the inhibition effect of 2,2'-(2-Hydroxyethylimino)bis[N-(alphaalphadimethylphenethyl)-N-methylacetamide] on the corrosion behavior of mild steel in the $1 \mathrm{M} \mathrm{HCl}$ was studied by using weight loss and electrochemical methods and the experimentally observed inhibition efficiency was explained theoretically using quantum chemical calculations. The following points emphasized the conclusion of the present work:

1. 2,2'-(2-Hydroxyethylimino)bis[N-(alphaalphadimethylphenethyl)-N-methylacetamide] showed an excellent inhibition effect on corrosion of mild steel in $1 \mathrm{M} \mathrm{HCl}$ solution, which was concentration and temperature dependent.

2. The inhibition performance can be attributed to the adsorption of this molecule on mild steel surface to form a protective film.

3. The inhibition behavior obtained from electrochemical studies showed the same trend as noticed in the weight loss studies. The inhibition efficiency increased with the increase in inhibitor concentration. The adsorption mechanism involved both physisorption and chemisorption.

4. The potentiodynamic polarization curves indicated that the inhibitor act as a mixed type of inhibitor with properties more cathodic in nature.

5. The adsorption of inhibitor on the metal surface followed Langmuir adsorption isotherm and the $\Delta \mathrm{G}_{\text {ads }}$ value calculated from adsorption studies had negative sign, indicating that the adsorption is spontaneous.

6. Determination of PZC indicated the formation of chloride bridge between protonated form of inhibitor molecules and metal surface.

7. Different computational methods were correlated to the inhibitive effect of 2,2'-(2-Hydroxyethylimino) bis [N-(alphaalpha-dimethylphenethyl)-Nmethylacetamide] .

8. The Fukui function results indicated that the preferred sites for both nucleophilic and electrophilic attack were nitrogen, oxygen, and some carbon atoms. The results also show that electronic factors, such as electron density, often have a greater influence in determining a good corrosion inhibitor than geometric factors of cases where more of the inhibitor is in contact with the metal surface. Therefore, even if a molecule has less planar geometry, it manages to have most of its electron donor centers in contact with the metal surface, it would have greater inhibition efficiency than a molecule that is planar but has less electron density.

9. The quantitative structure activity relationship (QSAR) approach has provided a good indication that an optimum of some quantum chemical parameters is required for good correlation with experimentally determined inhibition efficiency of the inhibitor.

\section{Acknowledgements}

The authors gratefully acknowledge the respective management and universities for providing sufficient facilities to carry out this research work and financial support.

\section{References}

1. Barros IBDB, Kappel MAA, Santos PMD, Junior VFDV, D’Elia E, Bastos IN. The inhibitory action of Bauhinia purpurea extracts on the corrosion of carbon steel in sulfuric acid medium. Mater Res. 2016;19:187-94.

2. Ballesteros AF, Gomes JAP, Bott IS. Corrosion evaluation of SAW Welded API 5L X-80 Joints in H2S-containing solution. Mater Res. 2015;18:417-26. 
3. Souza ECDCAD, Ripper BA, Perrone D, D’Elia E. Roasted coffee extracts as corrosion inhibitors for mild steel in HCL solution. Mater Res. 2016;19:1276-85.

4. Ochoa N, Bello M, Sancristóbal J, Balsamo V, Albornoz A, Britoc JL. Modified cassava starches as potential corrosion inhibitors for sustainable development. Mater Res. 2013;16:1209-19.

5. Gerengi H, Goksu H, Slepski P. The inhibition effect of mad Honey on corrosion of 2007-type aluminium alloy in 3.5\% $\mathrm{NaCl}$ solution. Mater Res. 2014;17:255-64.

6. Danaee I, Ghasemi O, Rashed GR, RashvandAvei M, Maddahy MH. Effect of hydroxyl group position on adsorption behavior and corrosion inhibition of hydroxybenzaldehyde Schiff bases: electrochemical and quantum calculations. J Mol Struct. 2013;1035:247-59.

7. Rocha JCD, Gomes JADCP, D'Elia E. Aqueous extracts of mango and orange peel as green inhibitors for carbon steel in hydrochloric acid solution. Mater Res. 2014;17:1581-7.

8. Santana PMBD, Meira M, Tentardini EK. Effects of adding some natural substances to biodiesel to control its effect on carbon steel corrosion. Mater Res. 2015;18:164-9.

9. Ghasemi O, Danaee I, Rashed GR, RashvandAvei M, Maddahy MH. The inhibition effect of synthesized 4-Hydroxybenzaldehyde1,3propandiamine on the corrosion of mild steel in $1 \mathrm{M} \mathrm{HCl}$. J Mater Eng Perform. 2013;22:1054-63.

10. Barreto LS, Tokumoto MS, Guedes IC, Melo HGD, Amado F, Capelossi VR. Study and assessment of the efficiency of the cocoa bark extracted from the theobroma cacao as an inhibitor of the corrosion of carbon steel in substitution of benzotriazole. Mater Res. In press 2018;21. http://dx.doi.org/10.1590/19805373-MR-2016-0309.

11. Santos ADM, Almeida TFD, Cotting F, Aoki IV, Melo HGD, Capelossi VR. Evaluation of castor bark powder as a corrosion inhibitor for carbon steel in acidic media. Mater Res. 2017;20:492505.

12. Sivakumar V, Velumani K, Rameshkumar S. Colocid dye - a potential corrosion inhibitor for the corrosion of mild steel in acid media. Mater Res. In press 2018;21. http://dx.doi. org/10.1590/1980-5373-MR-2017-0167.

13. Barros IBD, Custódio DL, Andrade MCD, Veiga VFD Jr, Silva AJD No, Bastos IN. The Inhibitory Action of Aniba Canelilla (H. B. K.) Mez. Extracts on the corrosion of carbon steel in hydrochloric acid medium. Mater Res. In press 2018;21(5). http://dx.doi.org/10.1590/1980-5373-MR-2017-0786.

14. Fergachi O, Benhiba F, Rbaa M, Touir R, Ouakki M, Galai $\mathrm{M}$, et al. Experimental and theoretical study of corrosion inhibition of mild steel in $1.0 \mathrm{M} \mathrm{HCl}$ Medium by $2(-4$ (hloro phenyl-1H- benzo[d]imidazol)-1-yl)phenyl)methanone. Mater Res. In press 2018;21. http://dx.doi.org/10.1590/1980-5373MR-2017-1038.

15. Santos EDCD, Cordeiro R, Santos MD, Paulo Rogério Pinto Rodrigues PRP, Singh A, D'Elia E. Barley agro-industrial residues as corrosion inhibitor for mild steel in $1 \mathrm{~mol} \mathrm{L-1}$ $\mathrm{HCl}$ solution. Mater Res. In press 2019;22(2). http://dx.doi. org/10.1590/1980-5373-MR-2018-0511.

16. Hoseinzadeh AR, Danaee I, Maddahy MH. Thermodynamic and adsorption behaviour of vitamin $\mathrm{B} 1$ as a corrosion inhibitor for AISI 4130 steel alloy in $\mathrm{HCl}$ solution. Z Phys Chem. 2013;227:403-18.

17. Zhang XY, Kang QX, Wang Y. Theoretical study of N-thiazolyl2-cyanoacetamide derivatives as corrosion inhibitor for aluminum in alkaline environments. Comput Theor Chem. 2018;1131:2532.

18. Edison TNJI, Atchudan R, Pugazhendhi A, Lee YR, Sethuraman MG. Corrosion inhibition performance of spermidine on mild steel in acid media. J Mol Liq. 2018;264:483-9.

19. RameshKumar S, Danaee I, RashvandAvei M, Vijayan M. Quantum chemical and experimental investigations on equipotent effects of $(+) \mathrm{R}$ and $(-) \mathrm{S}$ enantiomers of racemic amisulpride as eco-friendly corrosion inhibitors for mild steel in acidic solution. J Mol Liq. 2015;212:168-86.

20. Farahati R, Ghaffarinejad A, Mousavi-Khoshdel SM, Rezania J, Shockravi A. Synthesis and potential applications of some thiazoles as corrosion inhibitor of copper in $1 \mathrm{M} \mathrm{HCl}$ : experimental and theoretical studies. Prog Org Coat. 2019;132:417-28.

21. Abdallah M, Gad EAM, Sobhi M, Al-Fahemi JH, Alfakeer MM. Performance of tramadol drug as a safe inhibitor for aluminum corrosion in $1.0 \mathrm{M} \mathrm{HCl}$ solution and understanding mechanism of inhibition using DFT. Egyptian Journal of Petroleum. 2019;28:173-81.

22. Ebenso EE, Eddy NO, Odiongenyi AO. Adsorption and inhibitive properties of clarithromycin for the corrosion of $\mathrm{Zn}$ in 0.01 to 0.05 M H2SO4. Port Electrochem Acta. 2009;27:713-24.

23. Eddy NO, Ebenso EE. Adsorption and quantum chemical studies on cloxacillin and halides for the corrosion of mild steel in acidic medium. Int J Electrochem Sci. 2010;5:731-50.

24. El-Naggar MM. Corrosion inhibition of mild steel in acidic medium by some sulfa drugs compounds. Corros Sci. 2007;49:2226-36.

25. Morad MS. Inhibition of iron corrosion in acid solutions by cefatrexyl: behavior near and at the corrosion potential. Corros Sci. 2008;50:436-48.

26. Founda AS, Mostafa HA, El HM. Antibacterial drugs as inhibitors for the corrosion stainless steel type $304 \mathrm{in} \mathrm{HCl}$ solution. J Appl Electrochem. 2010;40:163-73.

27. Abdallah M. Rhodanine azosulpha drugs as corrosion inhibitors for corrosion of 304 stainless steel in hydrochloric acid solution. Corros Sci. 2002;44:717-28.

28. El-Naggar MM. Corrosion inhibition of mild steel in acidic medium by some sulfa drugs compounds. Corros Sci. 2007;49:2226-36.

29. Abdallah M. Antibacterial drugs as corrosion inhibitors for corrosion of aluminium in hydrochloric solution. Corros Sci. 2004;46:1981-96.

30. Shanbhag AV, Venkatesha TV, Prabhu RA, Kalkhambkar RG, Kulkarni GM. Corrosion inhibition of mild steel in acidic medium using hydrazide derivatives. J Appl Electrochem. 2008;38:279-87.

31. Vaszilcsin N, Ordodi V, Alexandra Borza A. Corrosion inhibitors from expired drugs. Int J Pharm. 2012;431:241-4.

32. Geethamani P, Kasthuri PK. The inhibitory action of expired asthalin drug on the corrosion of mild steel in acidic media: A comparative study. Journal of the Taiwan Institute of Chemical Engineers. 2016;63:490-9.

33. Singh P, Chauhan DS, Chauhan SS, Singh G, Quraishi MA. Chemically modified expired Dapsone drug as environmentally benign corrosion inhibitor for mild steel in sulphuric acid useful for industrial pickling process. J Mol Liq. 2019;286:110903.

34. Singh AK, Pandey AK, Banerjee P, Saha SK, Singh G. Ecofriendly disposal of expired anti-tuberculosis drug isoniazid and its role in the protection of metal. J Environ Chem Eng. 2019;7:102971.

35. Dohare P, Chauhan DS, Sorour AA, Quraishi MA. DFT and experimental studies on the inhibition potentials of expired Tramadol drug on mild steel corrosion in hydrochloric acid. Materials Discovery. 2017;9:30-41.

36. Anaee RA, Tomi IHR, Abdulmajeed MH, Naser SA, Kathem MM. Expired etoricoxib as a corrosion inhibitor for steel in acidic solution. J Mol Liq. 2019;279:594-602.

37. Gholami M, Danaee I, Maddahy MH, RashvandAvei M. Correlated ab initio and electroanalytical study on inhibition behavior of 2-mercaptobenzothiazole and its thiole-thione tautomerism effect for the corrosion of steel (API 5 L X52) in sulphuric acid solution. Ind Eng Chem Res. 2013;52:14875-89.

38. Aloysius A, Ramanathan R, Christy A, Baskaran S, Antony N. Experimental and theoretical studies on the corrosion inhibition of vitamins - Thiamine hydrochloride or biotin in corrosion of mild steel in aqueous chloride environment. Egyptian Journal of Petroleum. 2018;27:371-81. 
39. Gholivand K, Kahnouji M, Maghsoud Y, Masumian E, Hosseini M. A theoretical study on the coordination behavior of some phosphoryl, carbonyl and sulfoxide derivatives in lanthanide complexation. J Mol Model. 2018;24:328.

40. Nowroozi A, Masumian E. Comparative study of $\mathrm{NH} \cdots \mathrm{O}$ and $\mathrm{NH} \cdots \mathrm{S}$ intramolecular hydrogen bonds in $\beta$-aminoacrolein, $\beta$-thioaminoacrolein and their halogenated derivatives by some usual methods. Struct Chem. 2017;28:587-96.

41. Amin MA, Abd El-Rehim SS, Sherbini EEF, Bayoumi RS. The inhibition of low carbon steel corrosion in hydrochloric acid solutions by succinic acid Part I. Weight loss, polarization, EIS, PZC, EDX and SEM studies. Electrochim Acta. 2007;52:3588600 .

42. Hosseini M, Mertens SFL, Ghorbani M, Arshadi MR. Asymmetrical Schiff bases as inhibitors of mild steel corrosion in sulphuric acid media. Mater Chem Phys. 2003;78:800-8.

43. Doner A, Solmaz R, Ozcan M, Kardas G. Experimental and theoretical studies of thiazoles as corrosion inhibitors for mild steel in sulphuric acid solution. Corros Sci. 2011;53:2902-13.

44. Danaee I, Gholami M, RashvandAvei M, Maddahy MH. Quantum chemical and experimental investigations on inhibitory behavior of amino-iminotautomeric equilibrium of 2-aminobenzothiazole on steel corrosion in $\mathrm{H} 2 \mathrm{SO} 4$ solution. J Ind Eng Chem. 2015;26:81-94.

45. Hoseinzadeh AR, Danaee I, Maddahy MH. Thermodynamic and adsorption behaviour of medicinal nitramine as a corrosion inhibitor for AISI steel alloy in $\mathrm{HCl}$ solution. J Mater Sci Technol. 2013;29:884-92.

46. Benabdellah M, Touzani R, Aouniti A, Dafali A, El Kadiri $\mathrm{S}$, Hammoutia B, et al. Inhibitive action of some bipyrazolic compounds on the corrosion of steel in $1 \mathrm{M} \mathrm{HCl}$ : Part I: Electrochemical study. Mater Chem Phys. 2007;105:373-9.

47. Karimi A, Danaee I, Eskandari H, RashvanAvei M. Adsorption isotherm and inhibition effect of a synthesized di-(mFormylphenol)-1,2-cyclohexandiimine on corrosion of steel X52 in HCl solution. J Cent South Univ. 2016;23:249-57.

48. Musa AY, Kadhum AAH, MohamadAB, Takriff MS. Experimental and theoretical study on the inhibition performance of triazole compounds for mild steel corrosion. Corros Sci. 2010;52:3331-40.

49. Prabhu RA, Venkatesha TV, Shanbhag AV, Praveen BM, Kulkarni GM, Kalkhambkar RG. Quinol-2-thione compounds as corrosion inhibitors for mild steel in acid solution. Mater Chem Phys. 2008;108:283-9.

50. Ghasemi O, Danaee I, Rashed GR, RashvandAvei M, Maddahy MH. Inhibition effect of a synthesized N,N'-bis(2hydroxybenzaldehyde)-1,3-propandiimine on corrosion of mild steel in HCl. J Cent South Univ. 2013;20:301-11.

51. Oguzie EE, Unaegbu C, Ogukwe CN, Okolue BN, Onuchukwu AI. Inhibition of mild steel corrosion in sulphuric acid using indigo dye and synergistic halide additives. Mater Chem Phys. 2004;84:363-8.

52. Popova A, Christov M. Inhibitive properties of quaternary ammonium bromides of Ncontaining heterocycles on acid mild steel corrosion. Journal of the University of Chemical Technology and Metallurgy. 2008;43:37-47.

53. Ma H, Chen S, Yin B, Zhao S, Liu X. Preparation and hot corrosion behaviour of an $\mathrm{Al}$-gradient $\mathrm{NiCoCrAlYSiB}$ coating on a Ni-base super alloy. Corros Sci. 2003;45:867-82.

54. Solmaz R, Mert ME, Kardas G, Yazici B, Erbil M. Adsorption and corrosion inhibition effect of 1,1'-thiocarbonyldiimidazole on mild steel in $\mathrm{H} 2 \mathrm{SO} 4$ solution and synergistic effect of iodide ion. Wuli Huaxue Xuebao. 2008;24:1185-91.

55. Yurt A, Bereket G, Kivrak A, Balaban A, Erk B. Effect of schiff bases containing pyridyl group as corrosion inhibitors for low carbon steel in 0.1 M HCl. J Appl Electrochem. 2005;35:102532 .
56. Quraishi MA, Rawat J, Ajmal M. Dithiobiurets: a novel class of acid corrosion inhibitors for mild steel. J Appl Electrochem. 2000;30:745.

57. Ramesh Saliyan V, Adhikari AV. Quinolin-5-ylmethylene-3-\{[8 (trifluoromethyl)quinolin-4-yl]thio propanohydrazide as an effective inhibitor of mild steel corrosion in $\mathrm{HCl}$ solution. Corros Sci. 2008;50:55-61.

58. Karimi A, Danaee I, Eskandari H, RashvanAvei M. Electrochemical Investigations on the Inhibition Behavior and Adsorption Isotherm of Synthesized di(Resacetophenone)1,2 cyclohexandiimine Schiff base on the Corrosion of Steel in $1 \mathrm{M} \mathrm{HCl}$. Prot Met Phys Chem Surf. 2015;51:899-907.

59. Obot IB, Obi-Egbedi NO, Umoren SA. Antifungal drugs as corrosion inhibitors for aluminium in $0.1 \mathrm{M} \mathrm{HCl}$. Corros Sci. 2009;51:1868-75.

60. Popova A, Sokolova E, Raicheva S, Christov M. AC and DC study of the temperature effect on mild steel corrosion in acid media in the presence of benzimidazole derivatives. Corros Sci. 2003;45:33-58.

61. Solmaz R, Kardas G, Culha M, Yazici B, Erbil M. Investigation of adsorption and inhibitive effect of 2-mercaptothiazoline on corrosion of mild steel in hydrochloric acid media. Electrochim Acta. 2008;53:5941-52.

62. Tang L, Li X, Si Y, Mu G, Liu GH. Effects of sintering process and Mn-doping on microstructure and piezoelectric properties of $\mathrm{Pb}((\mathrm{Zn} 1 / 3 \mathrm{Nb} 2 / 3) 0.20(\mathrm{Zr} 0.47 \mathrm{Ti} 0.53) 0.80) \mathrm{O} 3$ system. Mater Chem Phys. 2006;95:26-70.

63. Solmaz R, Kardas G, Yazici B, Erbil M. Adsorption and corrosion inhibitive properties of 2-amino-5-mercapto-1,3,4thiadiazole on mild steel in hydrochloric acid media. Colloids Surf A Physicochem Eng Asp. 2008;312:7-17.

64. Zhan CC, Nichols JA, Dixon DA. Electron affinity, electronegativity, hardness, and electron excitation energy: molecular properties from density functional theory orbital energies. J Phys Chem A. 2003;107:4184-95.

65. Jafari H, Danaee I, Eskandari H, RashvandAvei M. Electrochemica and quantum chemical studies of N,N-bis(4-hydroxybenzaldehyde)2,2-dimethylpropandiimine Schiff base as corrosion inhibitor for low carbon steel in $\mathrm{HCl}$ solution. J Environ Sci Health Part A Tox Hazard Subst Environ Eng. 2013;48:1628-41.

66. Liu S, Parr RG. Consequences for exchange energy density functional of exponentially decaying nature of atomic electron densities. J Comput Chem. 1999;20:2-11.

67. Gomez B, Likhanova NV, Dominguez Aguilar MA, Olivares O, Hallen JM, Martinez-Magadan JM. Theoretical study of a new group of corrosion inhibitors. J Phys Chem A. 2005;109:8950-7.

68. Chattaraj PK, Sarkar U, Roy DR. Electrophilicity index. Chemical Reviews. 2006;10(2065):2065-91.

69. Liu SB. In chemical reactivity theory: a density functional view. Boca Raton: Taylor and Francis; 2009.

70. Jafari H, Danaee I, Eskandari H, RashvandAvei M. Electrochemica and theoretical studies of adsorption and corrosion inhibition of N,N'-Bis(2-hydroxyethoxyacetophenone)-2,2-dimethyl1,2-propanediimine on low carbon steel (API 5L Grade B) in acidic solution. Ind Eng Chem Res. 2013;52:6617-32.

71. Obot IB, Obi-Egbedi NO. Fluconazole as an inhibitor for aluminium corrosion in $0.1 \mathrm{M} \mathrm{HCl}$. Colloids Surf A Physicochem Eng Asp. 2008;330:207-12.

72. Obot IB, Obi-Egbedi NO. An interesting and efficient green corrosion inhibitor for aluminium from extracts of Chlomolaena odorata L. in acidic solution. Curr Appl Phys. 2010;40:1977-84.

73. Arslan T, Kandemirli F, Love I, Ebenso EE, Alemu H. Quantum chemical studies on the corrosion inhibition of some sulphonamides on mild steel in acidic medium. Corros Sci. 2009;51:35-47.

74. Gomez-Zavaglia A, Kaczor A, Cardoso AL, Teresa MVD, Melo $\mathrm{P}$, Fausto R. Unusual photochemical $\mathrm{C}-\mathrm{N}$ bond cleavage in the novel Methyl 2-Chloro-3-methyl-2Hazirine-2-carboxylate. J Phys Chem A. 2006;110:8081-92. 
75. Gazquez JL, Cedillo A, Vela A. Electrodonating and electroaccepting powers. J Phys Chem A. 2007;111:1966-70.

76. Ebenso EE, Arslan T, Kandemirli F, Caner N, Love I. Quantum chemical studies of some rhodanine azosulpha drugs as corrosion inhibitors for mild steel in acidic medium. Int J Quantum Chem. 2010;110:1003-18.

77. Abboud Y, Abourriche A, Berrada MS, Charrouf M, Bennamara A, Al Himidi N, et al. 2,3-Quinoxalinedione as a novel corrosion inhibitor for mild steel in $1 \mathrm{M} \mathrm{HCl}$. Mater Chem Phys. 2007;105:1-5.

78. Abdallah M, El-Naggar MM. $\mathrm{Cu}+2$ cation+3,5-dimethyl pyrazole mixture as a corrosion inhibitor for carbon steel in sulfuric acid solution. Mater Chem Phys. 2001;71:291-8.

79. Goel R, Siddiqi WA, Ahamed B, Hussan J. Corrosion inhibition of mild steel in $\mathrm{HCl}$ by isolated compounds of Riccinus Communis(L.). E-J Chem. 2010;7:319-29.

80. Jafari H, Danaee I, Eskandari H, RashvandAvei M. Combined computational and experimental study on the adsorption and inhibition effects of $\mathrm{N} 2 \mathrm{O} 2$ schiff base on the corrosion of API 5L grade B steel in $1 \mathrm{~mol} / \mathrm{L} \mathrm{HCl}$. J Mater Sci Technol. 2014;30:239-52.

81. Lukovits I, Kalman E, Zucchi F. Model of the inhibition mechanism of thiourea compounds. Corrosion. 2001;57:915-9.
82. Obi-Egbedi NO, Obot IB. Anti-corrosive properties of xanthone on mild steel corrosion in sulphuric acid: experimental and theoretical investigations. Curr Appl Phys. 2010;11:382-92.

83. Hoseinzadeh AR, Danaee I, Maddahy MH, Rashvand Avei M. Taurine as a Green Corrosion Inhibitor for AISI 4130 Steel Alloy in Hydrochloric Acid Solution. Chem Eng Commun. 2014;201:380-402.

84. Eddy NO, Ita BI. Experimental and theoretical studies on the inhibition potentials of some derivatives of cyclopenta-1,3-diene for the corrosion of mild steel in $\mathrm{HCl}$ solutions. Int J Quantum Chem. 2011;6:3456-74.

85. Xia S, Qiu M, Yu L, Liu F, Zha H. Molecular dynamics and density functional theory study on relationship between structure of imidazoline derivatives and inhibition performance. Corros Sci. 2008;50:2021-9.

86. Lukovits I, Bako I, Shaban A, Kalman E. Polynomial model of the inhibition mechanism of thiourea derivatives. Electrochim Acta. 1998;43:131-6.

87. Lukovits I, Shaban A, Kalman E. Corrosion inhibitors: quantitative structure-activity relationships. Russ J Electrochem. 2003;39:177-81.

88. Khaled KF. Experimental and theoretical study for corrosion inhibition of mild steel in hydrochloric acid solution by some new hydrazine carbodithioic acid derivatives. Appl Surf Sci. 2006;252:4120-8. 